\title{
,Nur' Wahlkämpfer oder doch Populisten? Eine linguistische Analyse des populistischen Diskurses französischer Präsidentschaftskandidaten
}

Thea Göhring*

\begin{abstract}
Recently, populist movements are gaining more and more ground in western democracies. As a result, the term populism is applied more widely, thus losing its conceptual sharpness. This article therefore aims to identify characteristic features of populist discourse, as opposed to political discourse in general and election campaign communication in particular. This goal is pursued using a dual approach. Firstly, a conceptual discussion shall illustrate the current problems in defining populism. Secondly, the examination of the 2017 French presidential election campaign will provide empirical evidence for typical features of populist discourse. Within the theoretical framework of discourse analysis, a linguistic analysis of the TV show 15 minutes pour convaincre will reveal the main characteristics of populist discourse used by the top candidates Fillon, Hamon, Le Pen, Macron and Mélenchon. Based on these results, different forms of populism will be distinguished. Their common core leads to a multi-faceted definition of populism combining form and content (as follows): Populism is a phenomenon which is based on the appeal to the 'people' and anti-elitism as well as on an antagonistic logic, highly contrasting with the agonal character of political discourse.
\end{abstract}

Key words: discourse analysis, election campaign, France, political discourse, populism

\footnotetext{
* Rheinische Friedrich-Wilhelms-Universität Bonn, Institut für Klassische und Romanische Philologie (Abteilung für Romanistik); tgoehring@uni-bonn.de
} 


\section{Einleitung}

Populistische Bewegungen befinden sich in den westlichen Demokratien auf dem Vormarsch. Dies bezeugen nicht nur die Wahl Donald Trumps zum Präsidenten der USA im Jahr 2016, sondern auch zahlreiche Wahlerfolge populistischer Parteien in Europa. Die zunehmende Implantierung populistischer Bewegungen in die politische Landschaft und deren wachsende Anhängerschaft in weiten Teilen der Bevölkerung wird oftmals als Signal der in einer Krise befindlichen repräsentativen Demokratie oder auch als Bedrohung derselben wahrgenommen. ${ }^{1}$ Das Phänomen des Populismus sowie die wissenschaftliche Auseinandersetzung mit demselben sind nicht neu, haben jedoch in den letzten Jahren einen explosionsartigen Anstieg erfahren. Damit geht eine exzessive Verwendung des Begriffs Populismus bzw. Populist(en) einher, der einerseits ein analytischer, metapolitischer und linguistischer Begriff ist, andererseits aber auch in die politische Auseinandersetzung selbst einfließt. Dies wirft die Frage auf, inwiefern sich die wissenschaftliche Analyse der Objektebene überhaupt entziehen kann. In der politischen Auseinandersetzung fungiert der Begriff zumeist als stigmatisierende Fremdbezeichnung, zuweilen aber auch als Selbstbezeichnung: Wenn der politische Konkurrent den Gegner als Populisten bezeichnet, verwendet er eine Strategie der Ab- und ggf. der Ausgrenzung; wenn Politiker die Bezeichnung hingegen für sich reklamieren, geht dies meist mit einer Umdeutung und Umwertung des Begriffs einher. ${ }^{2}$ Aufgrund dieser verschiedenen Konzeptualisierungen des Begriffs und des Facettenreichtums des Phänomens ist Populismus im allgemeinen Sprachgebrauch ein schillernder, nicht trennscharfer Begriff. Um jedoch das Phänomen in seinem Kern und seinen verschiedensten Ausprägungen zu erfassen, bedarf es einer (sprach-)wissenschaftlichen Herangehensweise, die auf der Grundlage bereits errungener wissenschaftlicher Erkenntnisse sowie empirischer Untersuchungen eine (Arbeits-) Definition des Begriffs Populismus und Kriterien zu Merkmalen populistischen Sprachgebrauchs bereitstellt.

Dieses Ziel wird im vorliegenden Aufsatz verfolgt und exemplarisch anhand des französischen Präsidentschaftswahlkampfs 2017 erarbeitet. Die französische Präsidentschaftswahl 2017 steht beispiellos für eine Zuspitzung der politischen Lage und eine Umwälzung des Parteiensystems in Frankreich, die wesentlich durch das Erstarken des Populismus bedingt sind. Die als Rechtspopulistin par excellence geltende Marine Le Pen und der bekennende (Links-)Populist Jean-Luc Mélenchon erzielen historische Erfolge; zum ersten Mal in der Geschichte der Fünften Republik zieht kein Vertreter der etablierten Volksparteien (Parti Socialiste und Les Républicains) in den zweiten Wahlgang ein; die Zahl

\footnotetext{
1 Populismus kann jedoch auch als für die Demokratie notwendiges und der demokratischen Debatte zuträgliches Phänomen betrachtet werden (vgl. den von Decker 2006b herausgegebenen Sammelband Populismus. Gefahr für die Demokratie oder nützliches Korrektiv?).

2 Vgl. etwa folgende Äußerung Mélenchons in einem Interview mit L'Express: „Je n'ai plus du tout envie de me défendre de l'accusation de populisme. C'est le dégoût des élites - méritent-elles mieux? Qu'ils s'en aillent tous! J'en appelle à l'énergie du plus grand nombre contre la suffisance des privilégiés. Populiste, moi? J'assume!" (Mélenchon 2010).
} 
der Enthaltungen und der ungültigen Stimmen erreicht einen neuen Rekord und neuer französischer Präsident wird Emmanuel Macron ${ }^{3}$ nach einem fulminanten Aufstieg (beinahe) aus dem Nichts ${ }^{4}$ mit der zum Zeitpunkt der Wahl gerade einmal ein Jahr alten Bewegung En Marche! im Rücken. ${ }^{5}$

Zum Verständnis und zur Erklärung dieser Entwicklungen vermag eine linguistische Analyse des politischen Sprachgebrauchs entscheidend beizutragen, denn „Sprache ist nicht nur irgendein Instrument der Politik, sondern überhaupt erst die Bedingung ihrer Möglichkeit“" (Girnth [2002] 22015, 1). Sollen Charakteristika des populistischen Sprachgebrauchs identifiziert werden, so besteht ein zentrales Anliegen darin, diese von Charakteristika des politischen Sprachgebrauchs abzugrenzen, und dies insbesondere vom politischen Sprachgebrauch im Kontext des Wahlkampfs. So wird hier folgende zweifache These vertreten: Zum einen verwenden nicht nur Politiker, die von Wissenschaftlern und/oder der Öffentlichkeit als Populisten bezeichnet werden und sich z.T. auch selbst Populisten nennen, sondern auch andere Politiker populistische Strategien, und dies insbesondere in Zeiten des Wahlkampfs; zum anderen sind zahlreiche Merkmale, die in der öffentlichen Debatte oder in der Forschung als typisch für den populistischen Sprachgebrauch gelten, lediglich typisch für den politischen Sprachgebrauch im Allgemeinen und für den Sprachgebrauch im Wahlkampf im Speziellen, nicht aber populistisch. Die Schwierigkeit der Abgrenzung resultiert in einer Überschneidung der Funktionen politischen und populistischen Sprachgebrauchs: ${ }^{6}$ Die zentrale - wenn auch bei weitem nicht die einzige - Funktion politischen Sprachgebrauchs im Wahlkampf ist die Persuasion, die zum Gewinn von Wählerstimmen und schließlich zum Machterwerb führen soll. Dieses Ziel ähnelt einem der Ziele der Populisten, nämlich den Zuspruch des Volkes zu erhalten. Dadurch kommen im Sprachgebrauch von Wahlkämpfern einerseits und von Populisten andererseits sprachliche Mittel und kommunikative Strategien zum Einsatz, deren Überschneidung, aber auch Abgrenzung voneinander es zu ermitteln gilt. Daher liegt der vorliegenden Untersuchung die Frage zugrunde, welche kommunikativen Stra-

\footnotetext{
3 Im ersten Wahlgang, der am 23.04.2017 stattfand, erzielten die Spitzenkandidaten folgende Ergebnisse: Emmanuel Macron (La République en Marche!) 24,01\%, Marine Le Pen (Front National, im Juni 2018 umbenannt in Rassemblement National) 21,30\%, François Fillon (Les Républicains) 20,01\%, Jean-Luc Mélenchon (La France Insoumise) 19,58\%, Benoît Hamon (Parti Socialiste) 6,36\%. Im zweiten Wahlgang, der am 07.05.2017 stattfand und für den sich die beiden Spitzenkandidaten qualifizierten, unterlag Marine Le Pen mit 33,90\% deutlich gegenüber Emmanuel Macron mit 66,10\%. Im ersten Wahlgang wurden 22,23\% Enthaltungen und 2,00\% ungültige Stimmen gezählt, im zweiten Wahlgang 25,44\% Enthaltungen und 8,59\% ungültige Stimmen (vgl. Ministère de l'Intérieur 2017).

${ }^{4}$ Mit einem Studium an der École Normale d'Administration (ENA) und dem Institut d'Études Politiques de Paris (IEP) hat Macron eine für französische Politiker typische Ausbildung durchlaufen. Jedoch war er als Investmentbanker bei Rothschild \& Co. auch außerhalb der Politik tätig und bis er 2014 zum Minister für Wirtschaft, Industrie und Digitales ernannt wurde den Franzosen weitgehend unbekannt. In nur drei Jahren und im Alter von 39 Jahren - und damit als jüngstes französisches Staatsoberhaupt seit Napoleon Bonaparte - gelang ihm der Sprung ins Präsidentenamt.

5 Emmanuel Macron gründete die Bewegung En Marche! am 6. April 2016 in Amiens, am 8. Mai 2017 erfolgte die Umbenennung in La République En Marche!, die Parteistatuten wurden am 17. August 2017 verabschiedet (vgl. La République En Marche! o.J.).

${ }^{6} \mathrm{Zu}$ Sprachfunktionen in der Politik s. Girnth ([2002] 22015, 46-50).
} 
tegien zum Einsatz kommen, wie diese versprachlicht werden und ob sie - einzeln oder in spezifischer Kombination - als Wesensmerkmale von Populismus fungieren können. Dabei lässt sich möglicherweise eine Typologie des Populismus entwerfen, nach der verschiedene Politiker verschiedenen Spielarten des Populismus zugeordnet werden können. Diese Frage soll auf der Grundlage eines Korpus empirisch und systematisch beantwortet werden. Dazu wird der Sprachgebrauch der fünf Spitzenkandidaten der französischen Präsidentschaftswahl 2017 verglichen, unabhängig davon, ob die Kandidaten gemeinhin als Populisten gelten oder nicht. Durch einen solchen vergleichenden und postulatsfreien Ansatz innerhalb einer dezidiert linguistisch-deskriptiven Untersuchung wird sich zeigen können, wie der jeweilige Sprachgebrauch charakterisiert ist und inwiefern er als populistisch klassifizierbar ist.

\section{Diskursanalyse}

Den theoretischen Rahmen für die vorliegende Untersuchung bildet die Diskursanalyse. Aus romanistischer Perspektive situiert sie sich - gemeinsam mit anderen Teilbereichen der Linguistik - in einer Linguistik des Sprechens, einer linguistique de la parole, wie sie Saussure ([1916] 2013, 72, 90) ablehnte, oder einer lingüisitica del hablar, wie sie Coseriu (1955-56, 31) forderte. Das heißt, dass sie die Systematik des Sprachgebrauchs, der sprachlichen Zeichen in ihrer Beziehung zu den Sprachbenutzern, untersucht und Sprache als soziales Phänomen auffasst. Daher verlangt sie im Sinne einer kulturwissenschaftlichen Linguistik den Einbezug vielfältiger nicht-sprachlicher „Umfelder“ (Bühler 1934, 154-168), der konkreten Kommunikationssituation sowie der Akteure in die linguistische Analyse. Neben dem Kontext ist auch der Kotext von essentieller Bedeutung in einer diskursanalytischen Untersuchung, da ein sprachliches Phänomen nicht isoliert, sondern stets im Äußerungszusammenhang analysiert wird. Zudem findet die Analyse aller Ebenen der Sprache - vom Wort über den Satz und den Text bis hin zu transtextuellen Strukturen - und ihres Zusammenspiels Eingang in die diskursanalytische Untersuchung.

Die Diskursforschung zeichnet sich durch überaus zahlreiche, teils konvergierende, teils divergierende Ansätze sowie durch eine Vielzahl von Diskursbegriffen aus. ${ }^{7}$ Die deutsche Romanistik ist geprägt von der Coseriu'schen Tradition der Diskursanalyse, auf der die Forschung zu Diskurs- und Texttraditionen gründet (vgl. Schlieben-Lange 1983; Koch 1997; Wilhelm 2001; Lebsanft und Schrott 2015b). Coseriu ([1988] ${ }^{2} 2007$, 75) situiert den Diskurs in seinem Drei-Ebenen-Modell des Sprechens auf der individuellen Ebene und unter dem Gesichtspunkt der Tätigkeit und definiert ihn so als eine

\footnotetext{
7 Für einen Überblick s. das von Wrana (2014) herausgegebene Wörterbuch sowie das von Angermuller et al. (2014) herausgegebene Handbuch zur interdisziplinären Diskursforschung; zur Geschichte des Diskursbegriffs s. Lebsanft und Schrott (2015a).
} 
von einem Individuum auf der Grundlage des expressiven Wissens in einer bestimmten Kommunikationssituation aktuell ausgeübte Tätigkeit, deren Produkt der Text ist: „en lo particular, [el hablar] es el discurso (el acto o la serie de actos) de tal individuo en tal oportunidad“ (Coseriu 1955-56, 31). In Coserius theoretischem Rahmen und in der darauf aufbauenden Forschungstradition der deutschsprachigen Romanistik wird davon ausgegangen, dass die diskursive Tätigkeit bestimmte Regelmäßigkeiten aufweist, die als Diskurs- oder Texttraditionen bezeichnet werden. Dem liegt die Annahme zugrunde, dass - obwohl jeder Text bzw. Diskurs individuell und in eine spezifische Äußerungssituation eingebettet ist - der Sprecher doch nicht in jeder Äußerungssituation etwas völlig Neues schafft, sondern an bekannte Traditionen anknüpft, die sich in Form von Mustern im Text manifestieren. Text- bzw. Diskurstraditionen werden von sogenannten Diskursgemeinschaften ausgeübt (s. dazu Schrott 2015), wobei die Diskurstraditionen auf einer Diskursgemeinschaft beruhen, diese aber nicht begründen (umgekehrt verhält es sich bei Sprachgemeinschaften, vgl. Coseriu [1988] 2007, 86). Text- bzw. Diskurstraditionen sind in einem Diskursuniversum verankert, worunter „Themen“ oder „Bezugssysteme“ des Redens zu verstehen sind, und erhalten in Abhängigkeit von diesem ihren Sinn (vgl. Coseriu [1980 ${ }^{3}$ 1994, 134).

In diesem Sinne kann in Bezug auf den vorliegenden Untersuchungsgegenstand von der Politik als Diskursuniversum und von Politikern als einer Diskursgemeinschaft gesprochen werden. Der hier zugrunde liegende Diskursbegriff definiert sich damit über eine soziale Praxis, über ein gemeinsames Handlungsfeld, und zwar das politische Handeln bzw. enger gefasst das Betreiben des Wahlkampfs. Das Ziel der vorliegenden Untersuchung besteht darin, die von den Politikern ausgeübten Diskurs- bzw. Texttraditionen zu identifizieren, indem regelmäßig wiederkehrende Sprachgebrauchsmuster bestimmt werden. Dabei werden diejenigen Diskurs- bzw. Texttraditionen in den Blick genommen, die als Spezifika des populistischen Diskurses aufgefasst werden können. Um diese zu ermitteln, bedarf es zunächst einer theoretischen Auseinandersetzung mit dem Phänomen des Populismus.

\section{Populismus}

\subsection{Begriffsbestimmung}

In dem Maß, in dem in den letzten Jahren populistische Bewegungen ihren Siegeszug durch die westlichen Demokratien fortsetzen, geraten ihr Politikverständnis und ihre Sprachverwendung in den Fokus von Politik- und auch Sprachwissenschaft. Die heterogene Konzeptualisierung des Begriffs Populismus nicht nur in der öffentlichen Debatte, sondern auch in der aktuellen Forschung macht eine Begriffsbestimmung zur theoretischen Erfassung des Phänomens sowie dessen Operationalisierung für die empirische Untersuchung erforderlich. 
Auch wenn der Begriff erst seit einigen Jahren Legion ist, ist seine Verwendung bereits seit gut einhundert Jahren attestiert. ${ }^{8}$ Etymologisch ist Populismus bekanntlich auf lat. Populus ,Volk' zurückzuführen; ausgehend von der Etymologie und der Wortbildung ${ }^{9}$ kann Populismus zunächst als, volksnahe Geisteshaltung oder politische Richtung' paraphrasiert werden. Dieser bereits in der Etymologie enthaltene Verweis auf das Volk spielt bis heute eine wesentliche Rolle in der Bestimmung des Begriffs.

In einem frühen begriffsgeschichtlichen und -theoretischen Beitrag ${ }^{10}$ nennt Worsley $(1969,244)$ in Anlehnung an Edward Shils zwei definitorische Merkmale von Populismus, die die verschiedenen Ansätze der Definition von Populismus bis heute prägen: zum einen die Forderung der Populisten, dass das Staatswesen auf dem Volkswillen beruhen solle, und zum anderen die Forderung einer unmittelbaren Beziehung zwischen dem Politiker und dem Volk unter Ausschluss von Institutionen. ${ }^{11}$ In der einflussreichen Definition Canovans $(1981,294)$ werden diese Merkmale abstrahiert und in dem Rekurs auf das ,Volk' und den Anti-Elitarismus resümiert: „All forms of populism without exception involve some kind of exaltation of and appeal to ,the people', and all are in one sense or another anti-elitist." Ein Vergleich verschiedener in der aktuellen Populismusforschung gängiger Definitionen (Decker [2000]22004, 22-23; Decker 2006a, 12; Hartleb 2014, 53; Mény und Surel 2002, 12-13; Mudde 2004, 543; Mudde und Rovira Kaltwasser 2012, 8; Priester 2007, 8; Priester 2012) zeigt, dass diese beiden Merkmale - der Rekurs auf das ,Volk' und der Anti-Elitarismus - ihr kleinster gemeinsamer Nenner sind. Den gängigen Definitionen folgend wird auch im vorliegenden Aufsatz die These vertreten, dass es sich bei diesen beiden Merkmalen um notwendige Bestandteile der Definition von Populismus handelt.

Der dem Populismus zugrunde liegende Volksbegriff ist dabei jedoch nicht identisch mit demjenigen, der demokratischen Verfassungen zugrunde liegt: ${ }^{12}$ Populisten konstru-

\footnotetext{
8 Im Französischen ist populiste erstmalig 1907 und populisme erstmalig 1912 belegt (vgl. TLFi, s.v. populiste, populisme). Zunächst diente der Begriff der Bezeichnung einer französischen Literaturströmung der ersten Hälfte des 20. Jahrhunderts sowie zweier politischer Strömungen des ausgehenden 19. Jahrhunderts, die US-amerikanische People's Party und die russischen Narodniki, die als „Exemplarfälle des modernen Populismus“ gelten können (Decker [2000] 22004, 23). Schließlich trat die abstrahierende Bedeutung ,politische Strömung' hinzu.

9 Populismus entsteht durch Derivation der gelehrten Basis populus mit dem Suffix -ismus, das ,in Bildungen mit Substantiven (meist Namen) eine Geisteshaltung oder politische Richtung, die von jemandem stammt, sich von jemandem herleitet, [kennzeichnet]“ (Duden Online, s.v. Populismus). Das gleiche Wortbildungsmuster liegt dem Begriff in anderen Sprachen zugrunde, so auch dem Französischen populisme.

${ }^{10}$ Es handelt sich um einen Beitrag zu dem von Ionescu und Gellner 1969 herausgegebenen Sammelband, der den Beginn der Populismusforschung markiert.

${ }^{11}$ Die erste Forderung ist auf das Rousseau'sche Konzept der volonté générale sowie die Idee der Volkssouveränität zurückzuführen und somit kein genuin populistisches Konzept, zumal alle demokratischen Verfassungen einen Verweis auf den Volkswillen enthalten. Die zweite Forderung steht für eine Schwächung der repräsentativen Demokratie zugunsten plebiszitärer Elemente und sieht sich etwa in einigen Kantonen der Schweiz in die Realität umgesetzt. Dies verdeutlicht die Schwierigkeit, Populismus mit einer antidemokratischen Haltung zu verbinden.

${ }^{12}$ Dies wird durch die einfachen Anführungszeichen signalisiert (,Volk $)$.
} 
ieren ein idealisiertes ,Volk ${ }^{\text {}}{ }^{13}$ das mit einer homogenen Stimme spricht und als dessen Sprachrohr die Populisten fungieren. Aus diesem Volksbegriff werden bestimmte Teile der Bevölkerung ausgeschlossen (weshalb mit Reisigl 2012 von einem synekdochischen Volksbegriff gesprochen werden kann, vgl. 3.2), so auch die als feindlich begriffenen Eliten. Neben dem Feindbild der Eliten werden zahlreiche weitere Feindbilder konstruiert und aus dem Volksbegriff ausgeschlossen, weshalb neben einer vertikalen auch von einer horizontalen Achse der Abgrenzung gesprochen werden kann (vgl. Priester 2012). Diese auch als Othering bezeichnete Strategie äußert sich in der dichotomischen Gegenüberstellung von Freund und Feind, eines wir vs. DIE ANDEREN.

Der Rekurs auf das ,Volk' und der Anti-Elitarismus sind, wenn auch nur minimale, so doch inhaltliche Merkmale von Populismus und zeugen daher von einer Auffassung von Populismus als Ideologie. ${ }^{14}$ Diese kontrastiert mit zahlreichen anderen Konzeptualisierungen von Populismus, etwa als Strategie oder Diskurspraxis mit dem Ziel der Machteroberung (Laclau [2005] 2007) oder als Syndrom (Wiles 1969; Priester 2017). Eine weitere Herangehensweise, die aus sprachwissenschaftlicher Perspektive von besonderem Interesse ist, ist die Definition von Populismus als Stil, als eine spezifische Form der Kommunikation oder Rhetorik (s. u.a. Aalberg et al. 2017; Jagers und Walgrave 2007; Taguieff 1997). Ausgehend von der Tatsache, dass Sprache konstitutiv für politisches Handeln ist, wird auch im vorliegenden Aufsatz die These vertreten, dass sich Vertreter des Populismus durch einen spezifischen Sprachgebrauch auszeichnen. Dieser kann mit Coseriu (vgl. 2.) als ein Bündel spezifischer Diskurstraditionen konzeptualisiert werden, die von den Sprechern, die über das entsprechende diskurstraditionelle Wissen verfügen, ausgeübt werden.

Diese Gegenüberstellung wirft die Frage auf, ob Populismus einer Ideologie im Sinne einer komplexen Weltanschauung gleichkommt oder ob er eher ein Kommunikationsstil, eine Art der Rhetorik ist. Hier wird die Ansicht vertreten, dass dies nicht als binäre Opposition, sondern als Kontinuum zwischen zwei Polen aufzufassen ist und Populismus sich folglich zwischen diesen beiden Polen ansiedelt. Er zeichnet sich also sowohl durch inhaltliche als auch durch formale Bestandteile aus, die zudem nicht unabhängig voneinander sind, sondern in einem komplexen Wechselspiel zueinander stehen. Diese Auffassung vertreten auch Hartleb (2014) - wie der Untertitel seiner Monographie Internationaler Populismus als Konzept. Zwischen Kommunikationsstil und fester Ideologie deutlich zum Ausdruck bringt - und Decker (2006a, 11): „Das Verhältnis von Form und Inhalt ist jedoch kein sich wechselseitig ausschließendes. Gerade der Populismus macht

\footnotetext{
${ }^{13}$ Um dieses nach innen und rückwärts gerichtete populistische Volkskonzept zu erfassen, propagiert Taggart (2000) den Terminus des heartland: „Populism tends to identify itself with an idealized version of its chosen people, and to locate them in a similarly idealized landscape. [...] Populism therefore has implicit within it a conception of heartland“" (Taggart 2000,3).

${ }^{14}$ Aufgrund der Tatsache, dass dieses ideologische Moment recht reduziert ist, wird Populismus in Anlehnung an Freeden (1996) auch als "thin-centered ideology“, als ,dünne Ideologie“ bezeichnet, worunter eine Ideologie mit wenigen Kernelementen zu verstehen ist, die je nach Ausprägung mit Elementen anderer Ideologien kombiniert werden können (vgl. Hartleb 2014; Mudde 2004; Priester 2012).
} 
deutlich, dass die Form, indem sie auf bestimmte inhaltliche Auffassungen zurückverweist, selbst ideologische Qualität annimmt. Dies gilt sowohl für die diskursive Form die Technik der Ansprache und Überzeugung - als auch für die organisatorische Form Aufbau und Funktionsweise einer populistischen Partei - [...].“

Vor diesem Hintergrund ist zu folgender Arbeitsdefinition von Populismus zu kommen: Populismus ist eine auf einer antagonistischen Logik beruhende Erscheinung, in deren Zentrum der Rekurs auf das synekdochisch konzeptualisierte Volk steht, dem die als feindlich begriffenen Eliten gegenüberstellt werden. Diese Arbeitsdefinition geht auf die oben geschilderte etymologische Basis und - mit dem Rekurs auf das, Volk' und dem Anti-Elitarismus - auf den Minimalkonsens in der Populismus-Forschung zurück. Diese gängigen Aspekte werden hier durch die These erweitert, dass sich Populismus durch eine antagonistische Logik auszeichnet, wobei die Form selbst, indem sie Ausdruck bestimmter ideologischer Auffassungen ist, zum Inhalt wird. Die einzelnen Elemente dieser Arbeitsdefinition werden in der folgenden Korpusanalyse empirisch überprüft.

\subsection{Populismus aus sprachwissenschaftlicher Sicht}

Nähert man sich dem Phänomen des Populismus aus sprachwissenschaftlicher Perspektive, so gilt es, für den Populismus typische Sprachgebrauchsmuster und die mit diesen Formen korrelierenden Funktionen zu identifizieren. Ansätze dazu finden sich bereits in manchen politikwissenschaftlichen Studien, etwa bei Decker ([2000] 22004, 35-36) und Hartleb (2014, 52). Im Folgenden sollen genuin sprachwissenschaftliche und insbesondere korpusbasierte Studien referiert werden, um aufzuzeigen, auf welches Wissen aufgebaut werden kann und wie die nachfolgende Korpusanalyse zur Erweiterung desselben beiträgt.

Reisigl $(2012,142)$ vertritt die These, dass sich die populistische Rhetorik durch zwei unverzichtbare Merkmale auszeichne: die Synekdoche und das argumentum ad populum. Die rhetorische Figur der Synekdoche des Typs totum pro parte liegt dem populistischen Volksbegriff zugrunde, d.h. der Kollektivbegriff Volk steht lediglich für einen Teil der Bevölkerung, wohingegen andere Bevölkerungsteile aus dem Volksbegriff ausgeschlossen werden (vgl. 3.1). Dem trugschlüssigen Argumentationsmuster des argumentum ad populum folgend geben die Populisten vor, den direkten Willen des Volkes auszuüben. Auf der Grundlage einer Analyse des Sprachgebrauchs der Freiheitlichen Partei Österreichs (FPÖ) erarbeitet Reisigl (2002, 166-174) zehn Merkmale populistischen Sprachgebrauchs:

1. Schwarz-Weiß-Malerei und Sündenbockkonstruktion

2. Komplexitätsreduktion

3. das Motto, kein Blatt vor den Mund zu nehmen

4. abwertende Beschimpfung politischer Gegner

5. Froschperspektivierung

6. die Suggestion, zum Volk zu gehören und Sprachrohr desselben zu sein 
7. pathetische Dramatisierung und Emotionalisierung

8. gebetsmühlenartige Wiederholung

9. kalkulierte Ambivalenz

10. Erlösungsverheißung durch die charismatische Führerfigur

Einige dieser Merkmale lassen sich in jeweils unterschiedlicher Kombination in anderen Studien wiederfinden. So finden etwa die an erster und zweiter Stelle genannten Prinzipien Eingang in die von Lebsanft $(2015,52)$ vertretene These, dass ein zentrales Merkmal des populistischen Diskurses darin bestehe, „komplexe Sachverhalte gezielt auf dilemmatische Fragestellungen zu reduzieren, deren Beantwortung den Wählern entsprechend leicht fallen soll“. Die Analyse eines im Vorfeld der Europawahlen 2014 geführten Interviews mit Marine Le Pen zeigt, dass der dem Slogan des FN bei den Europawahlen 2014 „Oui à la France, non à Bruxelles“ zugrunde liegende Antagonismus „es in der Tat erlaubt, sämtliche von Marine Le Pen in dem Le Monde-Interview vom 13.10.2014 formulierten Aussagen zu ordnen“ (Lebsanft 2015, 291).

Mit populistischem Sprachgebrauch in Frankreich befasst sich auch Alduy (2017, 345357), die im Vorfeld der Präsidentschaftswahl 2017 den Sprachgebrauch der présidentiables Marine Le Pen, François Fillon, Nicolas Sarkozy, Alain Juppé, François Hollande und Jean-Luc Mélenchon untersucht. Das der Arbeit zugrundeliegende Korpus setzt sich aus mehr als 1.350 Texten, die zwischen 2014 und 2016 produziert wurden, zusammen und umfasst insgesamt knapp 2,5 Millionen Token (vgl. Alduy 2017, 18). Ausgewertet wird das Korpus über einen lexikometrischen Zugang, der durch qualitative Analysen ergänzt wird. Ein Vergleich der Frequenz von Schlüsselbegriffen des populistischen Diskurses (peuple(s), nation, élite(s), caste, système, vérité) im Sprachgebrauch der einzelnen Kandidaten zeigt das Gewicht der Thematik im Diskurs der jeweiligen Kandidaten auf. Zudem wird die Konzeptualisierung des Begriffs peuple durch die einzelnen Kandidaten mittels der statistischen Identifizierung der „constellation lexicale“ des Wortes erschlossen. Alduy kommt zu dem Schluss, dass insbesondere Le Pen und Mélenchon, in geringerem Umfang auch Sarkozy, als populistisch gelten können, wobei sich der „populisme nationaliste et identitaire“ Le Pens radikal von dem „populisme révolutionnaire, social et universaliste" Mélenchons unterscheide (Alduy 2017, 357).

Jagers und Walgrave (2007) verfolgen das Ziel, mithilfe eines Index die Ausprägung von Populismus messbar zu machen, und setzen dies am Beispiel belgischer Parteien um. Ihrer Studie liegt die Definition von Populismus als "political communication style“ (so der Titel der Studie) zugrunde. Dieser Kommunikationsstil weise drei konstitutive Merkmale auf: die Referenz auf das Volk, Anti-Elitarismus und Ausgrenzungsstrategien. Auf der Grundlage dieser Kriterien werden drei Indices kalkuliert: people index, antiestablishment-index und exclusivity-index. Der people-index und der anti-establishmentindex setzen sich aus der Häufigkeit der Referenzen auf das Volk bzw. das Establishment (worunter Eliten, Staat und Medien verstanden werden) sowie der Intensität dieser Referenzen zusammen. Der exclusivity-index misst die Häufigkeit der Referenzen auf soziale Gruppen in Verbindung mit einer negativen Evaluation und deren Verhältnis zu Referen- 
zen auf soziale Gruppen in Verbindung mit einer neutralen oder positiven Evaluation. Die Korpusanalyse, der insgesamt 120 Fernsehsendungen von 1999 bis 2001 zugrunde liegen, zeigt, dass der rechtsextreme Vlaams Blok die höchsten Indices in allen drei Kategorien aufweist. Je nach Kombination der Indices werden abschließend vier Spielarten von Populismus unterschieden: complete populism (den einzig der Vlaams Blok vertritt), excluding populism, anti-elitist populism und empty populism.

Die Populismus-Definition von Jagers und Walgrave (2007) liegt auch Aalberg et al. (2017) zugrunde, einer systematischen, empirischen und kontrastiv für 24 europäische Länder angelegten Untersuchung von Populismus als Kommunikationsstil. Populistische Kommunikation wird hier im Hinblick auf drei Dimensionen untersucht: die populistischen Akteure als Produzenten, die Rolle der Medien und die Rezeption des populistischen Diskurses durch die Bevölkerung. Die zahlreichen länderspezifischen Studien resümierend kommen Aalberg et al. $(2017,10)$ hinsichtlich populistischen Sprachgebrauchs zu dem Schluss, dass sich dieser durch die von Jagers und Walgrave (2007) herausgearbeiteten Merkmale (Rekurs auf das Volk, Anti-Elitarismus, Ausgrenzung sozialer Gruppen) und darüber hinaus insbesondere durch Emotionalisierung, die Beschimpfung der Gegner und Sündenbockkonstruktion auszeichne (dies entspricht den von Reisigl genannten Merkmalen 1, 4 und 7).

Ein ähnliches Ziel wie Jagers und Walgrave (2007) verfolgt Scharloth (2017) und demonstriert dies am Beispiel der Alternative für Deutschland (AfD). Zur Beantwortung der Frage, ob eine Partei (mehr oder weniger) populistisch ist oder nicht, bestimmt Scharloth $(2017,3)$ drei Kriterien: „den spezifischen Rekurs auf das Volk, die Kritik an Eliten und Institutionen und den Stil der politischen Auseinandersetzung“. Die ersten beiden Kriterien stimmen mit denjenigen von Jagers und Walgrave (2007) überein, doch ist die korpuslinguistische Operationalisierung differenzierter. Von besonderem Interesse ist das dritte Kriterium. Carakteristisch für populistischen Sprachgebrauch sei ein „skandalisierender, polarisierender Politikstil“ (Scharloth 2017, 4); ein Kriterium, dass sich mit den von Reisigl genannten Merkmalen 1 und 7 deckt. Folgende sprachliche Phänomene sind laut Scharloth $(2017,4)$ Indizien eines populistischen Stils: Kommunikationsverben, die auf Konflikte verweisen, Intensivierer, skandalisierende Vokabeln und negativ wertende Adjektive. Vor dem Hintergrund einer Korpusanalyse von Wahlprogrammen und Pressemitteilungen sieben deutscher Parteien im Kontext der Landtagswahl in RheinlandPfalz 2016 kommt Scharloth $(2017,13)$ nicht nur zu dem Schluss, dass die AfD „wie keine andere Partei“ „skandalisiert und polarisiert“, sondern auch, dass „die Ergebnisse [es nahe legen], den Landesverband der AfD in Rheinland-Pfalz als populistisch zu kategorisieren."

Die Ergebnisse dieser Studien werfen die Frage auf, in welchem Verhältnis inhaltliche und formale (ideologische und sprachliche) Merkmale zueinander stehen und ob es sprachliche Kriterien gibt, die gewissermaßen als Alleinstellungsmerkmal populistischen Sprachgebrauchs fungieren und diesen von politischem Sprachgebrauch im Allgemeinen unterscheidbar machen. Die vorliegende Studie erweitert dabei die vorhandenen 
Erkenntnisse um folgende Aspekte: Der Rekurs auf das, Volk‘ und der Anti-Elitarismus werden nicht auf der Grundlage eines vorgegebenen Sets an Begriffen ermittelt, sondern die Vielfalt der verwendeten Referenzbegriffe wird induktiv aus dem Korpusmaterial erhoben; darüber hinaus werden die zugrunde liegenden Konzepte von Volk und Eliten durch eine Analyse der impliziten und expliziten Prädikationen in einem interpretativhermeneutischen Zugang erschlossen. Einen besonderen Beitrag leistet die vorliegende Studie insbesondere durch die Untersuchung des von allen referierten Ansätzen indirekt oder auch direkt behandelte, doch nie umfassend operationalisierte Prinzip der antagonistischen Logik (bei Reisigl Kriterium 1). Ziel ist es, zu zeigen, dass dieses nicht ein Prinzip unter vielen ist, sondern den Kern der populistischen Logik ausmacht.

\section{Korpus: Populismus und Massenmedien}

Als Korpus dient die Serie von TV-Interviews 15 minutes pour convaincre, die am 20. April 2017 ab 20:00 Uhr auf France 2 live ausgestrahlt und von David Pujadas und Léa Salamé moderiert wurde. Durch die Ausstrahlung in der prime time durch den größten öffentlich-rechtlichen Sender Frankreichs erreichte sie mit 4,7 Mio. Zuschauern ein relativ großes und breites Publikum. Die Sendung spielte eine Schlüsselrolle im Wahlkampf, da sie die letzte Gegenüberstellung aller Kandidaten nur drei Tage vor dem ersten Wahlgang darstellte und da sich während der Sendung ein terroristisches Attentat auf den Champs Elysées in Paris ereignete, das den Verlauf der Sendung maßgeblich beeinflusste. Die Sendung zeichnet sich außerdem durch eine gute Vergleichbarkeit der Kandidaten hinsichtlich der Länge der Beiträge, des Zeitpunkts, der Themen und des medialen Settings aus. Die Textsorte des TV-Interviews ist durch mündlichen, interaktionalen und spontansprachlichen Sprachgebrauch charakterisiert, der eine große Direktheit und Agonalität bewirkt. Die Sendung besteht aus elf Einzelinterviews und einem gemeinsamen Fazit aller Kandidaten. Untersucht werden die fünf Spitzenkandidaten François Fillon (LR), Benoit Hamon (PS), Marine Le Pen (FN), Emmanuel Macron (EM) und Jean-Luc Mélenchon (LFI). Die Dauer der untersuchten Videoausschnitte beträgt insgesamt eine Stunde und 48 Minuten. Die Transkription des Videos erfolgte mithilfe der Software ELAN (vgl. Brugman und Russel 2004) und umfasst 22.687 Tokens. ${ }^{15}$

Massenmediale Kommunikation ist heutzutage typisch für den Wahlkampf, doch ist zu vermuten, dass sie zudem den populistischen Diskurs begünstigt (vgl. Esser, Stępińska und Hopmann 2017), eine Entwicklung, für die Taguieff (2002) den Begriff des télépopu-

\footnotetext{
${ }^{15}$ Wenn nicht anders angegeben ist die Quelle der in der Analyse angeführten Belege stets das hier beschriebene Korpus. Folgende Transkriptionskonventionen wurden verwendet: [eckige Klammern] für Überschneidungen, MAJUSKELN für betonte Wörter oder Wortteile, (.) für Mikropausen und / für (Selbst- und Fremd-) Unterbrechungen. Wie im Fließtext markiert [...] Auslassungen und Kursivschrift dient der Hervorhebung der zur Diskussion stehenden Elemente.
} 
lisme geprägt hat. ${ }^{16}$ Nach Meyer (2006) ist grundsätzlich zwischen einer Logik der Medien und einer Logik der Politik zu unterscheiden. Die Logik der Medien beruhe auf zwei Regelsystemen - der Selektionslogik und der Präsentationslogik -, die dem Sensationsund Neuigkeitsbedürfnis der Zuschauer gehorchen, um möglichst hohe Einschaltquoten zu erzielen. Die Logik der Politik hingegen sei durch langsame Prozesse, vernunftgeleitete Reflexion und Objektivität gekennzeichnet. Die Medialisierung der Politik habe zur Folge, dass sich die Logik der Politik immer mehr der Logik der Medien angleiche. Da, wie Diehl (2012) darlegt, die Logik des Populismus ähnlichen Maßstäben folgt wie die Logik der Medien - Personalisierung, Komplexitätsreduktion, Dramatisierung, Emotionalisierung -, stellt sich die zweifache Frage, „ob es die Populisten sind, welche die Massenmedien besonders gut nutzen können, oder ob es die Massenmedien sind, die durch ihre Aufmerksamkeitsregeln Politiker und Politikerinnen dazu bringen, sich populistisch zu verhalten“ (Diehl 2012). Nicht zuletzt führt die knapp bemessene Sendezeit dazu, dass komplexe politische Inhalte nicht entfaltet, sondern auf kurze, einprägsame sound bites reduziert werden (vgl. Decker und Lewandowsky 2011, 345-346).

\section{Analyse}

Auf der Grundlage des Korpus werden in der folgenden Analyse Merkmale des populistischen Diskurses, gerade auch in Abgrenzung zum politischen Diskurs im Allgemeinen und zum Sprachgebrauch im Wahlkampf im Speziellen, herausgearbeitet. Diese Merkmale können als populistische Diskurstraditionen konzeptualisiert werden, die sich als Muster im Text manifestieren (vgl. 2). Auf der Grundlage der Arbeitsdefinition von Populismus (vgl. 3.1) lassen sich folgende Kriterien ermitteln, die in ihrer spezifischen Ausprägung und Kombination als Merkmale des populistischen Diskurses fungieren können:

1. Rekurs auf das Volk

a) Die Frequenz der Referenzen auf das Volk in seiner Gesamtheit

b) Die Form und die Frequenz der Referenzausdrücke für das Volk, v.a. peuple

c) Ein auf der Figur der Synekdoche beruhendes Volkskonzept

2. Anti-Elitarismus

a) Die Frequenz negativ wertender Prädikationen über die Eliten

b) Die Form und die Frequenz der Referenzausdrücke für die Eliten

3. Antagonistische Logik

\footnotetext{
${ }^{16}$ „Dans le télépopulisme on retrouve l'appel au peuple, mais d'une façon telle qu'il puise l'essentiel de son efficacité symbolique dans les ressources propres de l'espace médiatique et dans la compétence télégénique des leaders“" (Taguieff 2002, 119).
} 
a) Die Konzeptualisierung bestimmter Objekte als Antagonisten

b) Die Frequenz der Referenz auf Antagonisten

Das Korpus wird systematisch auf diese Kriterien hin untersucht, indem in einem ersten Schritt durch die Lektüre des Korpus alle relevanten Aussagen und Referenzausdrücke identifiziert werden und in einem zweiten Schritt die Frequenz derselben für jeden einzelnen Kandidaten erhoben wird. In einem dritten Schritt werden die zugrundeliegenden Konzepte von Volk und Eliten sowie die Konstruktion von Antagonismen durch eine Untersuchung der expliziten und impliziten Prädikationen erschlossen. Die Analyse ist somit primär qualitativ orientiert, wird jedoch durch quantitative Aspekte gestützt. Die Frequenzen wurden mithilfe der Software AntConc erhoben (vgl. Anthony 2016).

\subsection{Rekurs auf das Volk}

Eine besonders häufige Referenz auf das Volk kann als erstes Indiz für eine populistische Haltung fungieren. Tabelle 1 zeigt die Frequenz expliziter Referenzen auf das Volk in seiner Gesamtheit für die einzelnen Politiker, die dem ideologischen Spektrum folgend von links nach rechts aufgeführt sind. Es zeigt sich, dass Mélenchon und Le Pen mit Abstand am häufigsten auf das Volk referieren. Es folgen, in absteigender Reihenfolge, Hamon, Fillon und Macron.

\begin{tabular}{ccccc}
\hline Mélenchon & Hamon & Macron & Fillon & Le Pen \\
\hline 43 & 15 & 8 & 12 & 38 \\
\hline
\end{tabular}

Tabelle 1: Frequenz der Referenzen auf das Volk in seiner Gesamtheit

Zu der Häufigkeit der Referenz auf das Volk kommen als zweites Kriterium die verwendeten Referenzausdrücke hinzu. Diese geben Aufschluss über die Konzeptualisierung des Volks durch die jeweiligen Kandidaten, da durch die Verwendung eines Referenzausdrucks bereits eine implizite Prädikation über den Referenten erfolgt. Tabelle 2 zeigt die Substantive, mit denen die untersuchten Politiker am häufigsten auf das französische Volk referieren, sowie die Anzahl der jeweiligen Okkurrenzen.

\begin{tabular}{lccccc}
\hline & Mélenchon & Hamon & Macron & Fillon & Le Pen \\
\hline Français & 4 & 5 & 0 & 3 & 19 \\
peuple & 15 & 4 & 0 & 0 & 4 \\
citoyen(ne)(s) & 4 & 3 & 0 & 0 & 0 \\
concitoyens & 0 & 2 & 5 & 0 & 0 \\
compatriotes & 1 & 1 & 0 & 0 & 1 \\
\hline
\end{tabular}

Tabelle 2: Referenzausdrücke für das Konzept DAS FRANZÖsISCHE VolK 
Der am häufigsten verwendete Referenzausdruck ist das Gentonym Français. Als Derivation des Staatennamens France definiert dieser Begriff die Personengruppe, auf die referiert wird, über die Staatsangehörigkeit. Hervor sticht Le Pen, die den Begriff deutlich häufiger verwendet als die übrigen Politiker (zu den 19 Okkurrenzen kommen zudem 11 pronominale Wiederaufnahmen); Français ist das von Le Pen am häufigsten verwendete Substantiv im gesamten Korpus (zur Bedeutung dieses Begriffs für die Konzeptualisierung des Volks durch Le Pen vgl. infra).

Der am zweithäufigsten verwendete Referenzausdruck ist peuple, der als besonderes Indiz für eine populistische Haltung fungiert, da er - genau wie Populismus - auf lat. Populus ,Volk' zurückzuführen ist. Er wird von Mélenchon, Hamon und Le Pen verwendet. Mit 15 Okkurrenzen ist peuple das häufigste von Mélenchon verwendete Substantiv insgesamt; Le Pen verwendet den Begriff zwar deutlich seltener, doch dafür an exponierter Stelle (vgl. Beleg 8). Die hohe Frequenz des Begriffs peuple im Sprachgebrauch Le Pens und Mélenchons bestätigt Alduy $(2017,292)$ auf der Grundlage eines deutlich größeren und repräsentativeren Korpus: Bei Le Pen ist peuple das siebthäufigste Substantiv, bei Mélenchon steht es an neunter Stelle. Zum Vergleich: Bei Fillon rangiert es auf Platz 93, bei Juppé lediglich auf Platz 371 und bei Hollande auf Platz 313. Dass der Begriff peuple und mit ihm das Volk im Zentrum des jeweiligen Wahlkampfs von Mélenchon und Le Pen steht, bringen bereits ihre den Begriff peuple enthaltenden Slogans zum Ausdruck: Mélenchon macht unter dem Slogan La force du peuple Kampagne, Le Pen unter Au nom du peuple. Mit diesem Slogan greift Le Pen die in Frankreich übliche Eingangsformel gerichtlicher Entscheidungen, insbesondere von Urteilen, auf, wie sie im Code de procédure civile, Art. 454 festgehalten ist: „Le jugement est rendu au nom du peuple français“ (CPC, Art. 454). Doch nicht nur die Legislative, sondern auch die Exekutive bedient sich dieser Formel, und zwar in der sogenannten formule exécutoire (vgl. Lebsanft 2018, 35-36). ${ }^{17} \mathrm{Le}$ Pen verleiht damit der Gesamtheit ihres Wahlkampfs den Schein eines juristisch valablen Tuns.

Weitere in Tabelle 2 aufgeführte Referenzausdrücke für das Volk sind citoyen(ne)(s), concitoyens und compatriotes. Mit dem Begriff citoyen wird das Volk als politisch organisierte Gemeinschaft konzeptualisiert. Ähnlich verhält es sich mit dem Begriff concitoyen, der durch das Präfix con- zudem eine unverzichtbare Pluralität zum Ausdruck bringt, wodurch die zwischen den Bürgern bestehenden Beziehungen hervorgehoben werden. Compatriotes < lat. PATRIA ,Vaterland' hingegen betont die emotionale und genealogische Verbundenheit mit dem Land. ${ }^{18}$ Mit compatriotes wird das Volk somit als politisches und patriotisches Kollektiv konzeptualisiert, mit concitoyens hingegen als po-

\footnotetext{
${ }^{17}$ Lebsanft (2018) zeigt gewisse Parallelen zwischen dem Volksbegriff Le Pens und dem der französischen Gesetzestexte auf, macht in einer differenzierten Analyse aber zugleich deutlich, dass sich Le Pens Volksbegriff im Gegensatz zu dem juristischen über die ethnische Zugehörigkeit definiert, was der neue discours frontiste sogar nur noch bedingt zu dissimulieren scheine.

${ }^{18}$ In compatriotes lässt sich darüber hinaus ein Anknüpfen an die französische Nationalhymne als Symbol der französischen Identität sehen, deren erster Satz die ,Kinder des Vaterlands‘ adressiert: „Allons enfants de la Patrie, le jour de gloire est arrivé!“
} 
litisches und staatsbürgerliches Kollektiv (vgl. die Derivationen patriote, patriotisme vs. citoyen, citoyenneté) (vgl. Rigat 2010). Aufgrund der sehr niedrigen Frequenzen dieser Begriffe lassen diese jedoch keine repräsentativen Aussagen über die mit ihnen einhergehende Konzeptualisierung des Volks durch die einzelnen Kandidaten zu. ${ }^{19}$

Im Folgenden wird dargelegt, welche unterschiedlichen Konzepte des Volks die einzelnen Kandidaten entwerfen. Dazu wird zum einen auf die in Tabelle 2 aufgeführten Referenzausdrücke zurückgegriffen, zum anderen werden weitere Referenzausdrücke diskutiert und schließlich werden Attributionen und Prädikationen, die in Verbindung mit diesen auftreten, analysiert und implizite Inferenzen und Präsuppositionen offengelegt.

Mélenchon vertritt ein synekdochisches Volkskonzept, das sich über bestimmte soziale Gruppen sowie in radikaler Weise über das demos definiert. Der von ihm sehr häufig verwendete Referenzausdruck peuple steht totum pro parte nur für einen Teil der Gesamtbevölkerung, und zwar für sozial und wirtschaftlich niedriger gestellte Bevölkerungsgruppen, für den ,kleinen Mann'. Mélenchon nennt u.a.: classe moyenne, chômeur(s), blessés de la vie, morts dans la rue und pawvres. Das Eintreten für diese Bevölkerungsgruppen ist typisch für Politiker und Parteien des linken ideologischen Spektrums (so auch Hamon, vgl. infra). Ein auf diesen Bevölkerungsgruppen basierendes synekdochisches Volkskonzept kann somit als typisches Merkmal von Linkspopulismus betrachtet werden. Dass sich Mélenchon insbesondere für diese Bevölkerungsgruppen stark macht, illustriert Beispiel (1): Er polarisiert gegen diejenigen, die in Bezug auf Bildung und Beruf und damit auch Einkommen besser gestellt sind, und tritt für die anderen ein, die er schlicht als gens bezeichnet und durch das Adjektivattribut braves als rechtschaffen und anständig charakterisiert; ${ }^{20}$ in dem Relativsatz betont er zudem die niedrigen Ansprüche und Erwartungen der braves gens, womit eine implizite Handlungsaufforderung an die Politiker einhergeht, diese zu erfüllen, verbunden mit dem Vorwurf, dass sie bislang nicht erfüllt wurden:

(1) Mélenchon: Il faut que tout le monde ait son compte et pas seulement ceux qui ont fait les grandes études ou ceux qui ont des diplômes. Tant mieux, on est très content, eux aussi doivent avoir du travail. Mais aussi les braves gens qui veulent juste vivre de leur vie avec un salaire qui permet d'avoir une vie digne. Et moi, c'est à eux que je pense, voyez-vous?

Ein Alleinstellungsmerkmal Mélenchons ist die häufige Verwendung des Referenzausdrucks gens für das Volk (fünf Okkurrenzen im Vergleich zu keinen bei den übrigen Kandidaten). Auch mit diesem Begriff stellt Mélenchon den ,kleinen Mann', le tout-venant

\footnotetext{
${ }^{19}$ Auch die Frage einer ideologischen Konnotation der Begriffe - etwa, ob concitoyens überwiegend von der französischen Linken, compatriotes hingegen überwiegend von der französischen Rechten verwendet wird (vgl. Rigat 2010), - bedarf eines größeren Korpus als dem dieser Untersuchung zugrunde liegenden.

${ }^{20}$ Man vergleiche die Bedeutungsangaben für braves gens in einschlägigen Wörterbüchern: „gens honnêtes et bons“ (Grand Robert, s.v. gens), „Personnes recommandables, qui ont de la simplicité, de la droiture dans les manières" (TLFi, s.v. gens).
} 
des Français, in den Fokus seines Volkskonzepts. Mélenchons Volksbegriff definiert sich jedoch nicht nur über eine bestimmte soziale ,Schicht', sondern auch in radikaler Weise über das demos < gr. $\delta \tilde{\eta} \mu \circ$,Staatsvolk', d.h. über das Volk als Quelle der demokratischen Souveränität (dies stellt auch Alduy 2017, 293-294, 354-357 fest). Dies wurzelt in seiner Forderung, dass dem ,kleinen Mann' in der von ihm angestrebten Sechsten Republik mehr politische Macht zukommen solle (was mit einer Schwächung der Eliten einhergeht, vgl. 5.2). Der Frage der Moderatorin, ob das Land dadurch nicht unregierbar werde, entgegnet Mélenchon in (2) mit einer Umdeutung des Demokratie-Begriffs, ${ }^{21}$ aus der eine Haltung spricht, die nach Mudde $(2004,561)$ als „democratic extremism“ charakterisiert werden kann. Der Rekurs auf den Topos der Angst vor dem Volk, die er seinen Gegnern unterstellt, unterstreicht diese Haltung.

(2) Mélenchon: Mais jamais de la vie! Pourquoi serait-il ingouvernable? [...] C’est pas la pagaille permanente, c'est l'inverse. Mais les gens au pouvoir, vous comprenez, ça change la mentalité de tout le monde, de savoir que les gens peuvent se mettre au travail pour collecter le nombre de signatures qui permet d'organiser un référendum révocatoire. Mais enfin, les amis, ça s'appelle la démocratie! Faut pas avoir peur du peuple!

Die Figur des Politikers tritt dadurch völlig in den Hintergrund. So spricht Mélenchon nicht im Namen des Volkes (im Gegensatz zu Le Pen, vgl. Beleg 8), sondern fordert, dass das Volk selbst seine Stimme erhebe (vgl. Alduy 2017, 290). Seine Rolle sieht er konsequenterweise darin, den Prozess bis zum Inkrafttreten der Verfassung der Sechsten Republik zu begleiten und dann ,zu gehen'. ${ }^{22}$ Seine Person verschwindet hinter der Wichtigkeit der Sache, der er dient (vgl. Alduy 2017, 63-68). Der Aussage der Moderatorin Léa Salamé, dass er wie kaum ein anderer das de Gaulle'sche Ideal der rencontre d'un homme et d'un peuple verkörpere, weist er demnach auch entschieden zurück und stellt das Volk an erste Stelle:

(3) Salamé: [...][cette] rencontre d'un homme et d'un peuple, c'est tellement VOUS. $[\ldots]$

Mélenchon: [...] Vous êtes bien aimable de dire que c'est tout à fait moi, mais moi, je ne me représente pas les choses comme ça. Je pense que la force vient de l'intelligence collective.

Das Volkskonzept Hamons ähnelt in seinen Grundzügen demjenigen Mélenchons, doch in deutlich abgeschwächter Form. Als Politiker des linken ideologischen Spektrums vertritt auch Hamon einen Volksbegriff, der totum pro parte für den ,kleinen Mann` steht,

\footnotetext{
${ }^{21}$ Die den politischen Diskurs und die öffentliche Debatte zunehmend prägenden Forderungen der Populisten haben in Frankreich, aber auch in anderen Ländern, eine Debatte über den Demokratiebegriff ausgelöst, der - je nach ideologischer Gesinnung - vielfachen Umdeutungen unterworfen ist (vgl. auch Beleg 20).

22 „Une fois que la Constitution est votée, elle s'applique et donc, je m'en vais.“ (Mélenchon in On n'est pas couché, France 2, 22.09.2016, zit. nach Alduy 2017, 64).
} 
und auch er tritt insbesondere für sozial und wirtschaftlich schlechter gestellte Teile der Bevölkerung ein, etwa: salariés, les salariés sous 2.200 euros net par mois, quelqu'un qui est au smic oder auch diejenigen, die unter einem handicap oder unter discrimination leiden. Hamons Volksbegriff ist jedoch inklusiver als derjenige Mélenchons, da er sich auch für besser gestellte Gruppen wie die fonctionnaires oder auch für die générations qui viennent im Allgemeinen stark macht. Zudem polarisiert er deutlich weniger gegen die Eliten (vgl. dazu 5.2) und propagiert keinen democratic extremism.

Macron vertritt kein synekdochisches, sondern ein inklusives Volkskonzept. Dies kommt u.a. darin zum Ausdruck, dass er nicht gegen die Eliten polarisiert (vgl. 5.2) und dass er sich für diverse Bevölkerungsgruppen stark macht, die das ganze Spektrum der Gesellschaft abdecken: artisans, chômeurs, classes moyennes et populaires, classes moyennes et supérieures, élèves, enseignants, familles, jeunes filles et garçons, des jeunes ou des moins jeunes, propriétaires, travailleurs. Darüber hinaus verwendet er als Referenzausdrücke für das Volk in seiner Gesamtheit neben concitoyens (vgl. Tabelle 2) ausschließlich die Bezeichnungen chacune et chacun, toutes et tous und tout le monde. Diese Ausdrücke, die auf Indefinitpronomina basieren und wenn möglich zwischen femininem und maskulinem Geschlecht differenzieren, fungieren gewissermaßen als Allquantor und adressieren so eine möglichst breite Masse, heben aber zugleich auch jeden Einzelnen in dieser Gesamtheit hervor. Die daraus sprechende Haltung korreliert mit dem Slogan, unter dem Macron Kampagne macht: La Chance doit être une chance pour tous. Das folgende Beispiel illustriert exemplarisch dieses auf Inklusion und Gleichberechtigung basierende Volkskonzept Macrons:

(4) Macron: Il y a un cadre, le cadre de la République. Et il ne changera pas. Et donc dans ce cadre, dans ces règles, qui seront appliquées à toutes et tous, je veux $[\ldots]$.

Auch Fillons Volkskonzept ist nicht synekdochisch. Als Referenzausdrücke für das französische Volk verwendet er neben Français (vgl. Tabelle 2) auch communauté nationale, nation, population française und société française. Auffällig ist, dass er dadurch als einziger auf das Konzept der Nation abhebt. Die Tatsache, dass er keine spezifischen sozialen Gruppierungen erwähnt, lässt darauf schließen, dass er sich zwar einerseits für keine soziale Gruppierung besonders stark macht (im Gegensatz zu Mélenchon und Hamon), sich sein Volkskonzept aber auch nicht durch eine besondere Diversität auszeichnet (im Gegensatz zu demjenigen Macrons). Eine zunehmend populistische Haltung Fillons lässt sich im Laufe der Affaire Fillon ${ }^{23}$ konstatieren, in deren Zuge er sich zunächst als Opfer

\footnotetext{
${ }^{23}$ Als Affaire Fillon oder Penelopegate wird der politische Skandal um François Fillon bezeichnet, in dessen Kontext er u.a. der Veruntreuung öffentlicher Gelder bezichtigt wurde. Mehrere ab Ende Januar 2017 im Canard enchaîné erschienene Artikel werfen François Fillon vor, seine Frau Penelope Fillon und zwei seiner Kinder illegitimerweise auf staatliche Kosten beschäftigt zu haben. Auf juristische Vorermittlungen folgte am 1. März 2017 die offizielle Anklage gegen Fillon, die ihm im Wahlkampf die Unterstützung vieler Politiker kostete und ein Popularitätstief in den Meinungsumfragen bescherte. Fillon zog seine Kandidatur nicht zurück und schied im ersten Wahlgang aus dem Rennen um die französische Präsidentschaft aus.
} 
eines „assassinat politique“ bezeichnet (Fillon zit. nach Big Browser 2017) und dann das Volk zu seinem wahren Richter deklariert, dessen Urteil mit dem Wahlergebnis fallen werde (5). Diese Aussagen zeugen sowohl von einer Infragestellung juristischer Instanzen als auch von der Konstruktion einer direkten Beziehung zwischen sich und dem Volk.

(5) Fillon: Au-delà de la procédure judiciaire, c'est au peuple français et à lui seul que j'en appelle désormais (Fillon zit. nach Montvalon 2017).

Le Pens Volkskonzept ist wie dasjenige Mélenchons und Hamons synekdochisch. Auch sie stellt dem ,kleinen Mann' die als feindlich begriffenen Eliten gegenüber (vgl. 5.2). Auffällig ist jedoch, dass sie seltener einzelne Bevölkerungsgruppen nennt, sondern überwiegend die Begriffe Français und peuple verwendet, um auf das französische Volk zu referieren. Sie konzeptualisiert das Volk dadurch als homogene Einheit und suggeriert, dass sie die Gesamtheit der Franzosen adressiere, was sie in (6) zudem durch die Nennung der Einwohnerzahl explizit macht:

(6) Le Pen: Il y a 65 millions de Français qui attendent d'avoir un très bon avocat. [...] Je passe mes journées entières à les défendre et j'en suis toujours comblée d'ailleurs.

Jedoch stehen die Begriffe Français und peuple bei Le Pen nicht nur totum pro parte für den ,kleinen Mann', sondern auch für eine sich über die ethnische Zugehörigkeit definierende Gemeinschaft. Bevölkerungsgruppen anderer ethnischer Zugehörigkeit werden aus diesem Volksbegriff ausgeschlossen, weshalb es sich um einen exklusiven - im Gegensatz zu einem inklusiven - Volksbegriff handelt. Dieses sich über das etbnos $<$ gr. $气$ ध̈vos ,[fremdes] Volk, Volkszugehörige‘ definierende, exklusive Volkskonzept ist typisch für rechtspopulistische Bewegungen (vgl. Decker 2006a, 13-14). So schließt Le Pen etwa Migranten aus ihrem Volksbegriff aus (vgl. 5.3) und beabsichtigt zudem, den Erwerb der französischen Staatsangehörigkeit stärkeren Restriktionen zu unterwerfen, u.a. durch die Abschaffung des ius soli:

(7) Le Pen: Moi, je veux la suppression du droit du sol. Je pense que la nationalité française s'hérite ou se mérite.

Alleinstellungsmerkmal Le Pens ist darüber hinaus, dass sie sich in typisch populistischer Manier als Sprachrohr des Volkes inszeniert. Dies kommt besonders deutlich in ihrem Schlussstatement zum Ausdruck (8). In diesem greift sie nicht nur den Slogan Au nom du peuple auf (vgl. supra), sondern radikalisiert auch folgendes, in der französischen Verfassung verankertes Prinzip: „,...] Son principe [le principe de la République] est: gouvernement du peuple, par le peuple et pour le peuple. La souveraineté nationale appartient au peuple qui l'exerce par ses représentants et par la voie du référendum. [...]“ (Constitution, Art. 2-3). Die Radikalisierung besteht darin, dass laut Verfassung das Volk - repräsentiert durch die Abgeordneten der Republik - regiert, laut Le Pen aber sie allein dieses Volk repräsentiere. Ihrer Aussage liegen ein Absolutheitsanspruch und eine 
dichotomische Darstellungsweise zugrunde, durch die implizit den anderen Politikern vorgeworfen wird, nicht (immer) im Namen des Volkes zu handeln:

(8) Le Pen: Je ne ferai rien SANS le peuple français ou CONTRE lui. Ma campagne, c'est au nom du peuple que je la mène, eh bien, mon quinquennat, c'est au nom du peuple que je le mènerai aussi.

Zusammengenommen zeugen die Belege (7) und (8) von einem Amalgam der Konzepte von demos - dem Volk als Quelle der demokratischen Souveränität - und ethnos - dem Volk als ethnische Einheit -, das dem Volkskonzept Le Pens zugrunde liegt (zu diesem Schluss kommt auch Alduy 2017, 348).

Insgesamt hat die Analyse des Rekurses auf das Volk gezeigt, dass Mélenchon und Le Pen am häufigsten auf das Volk rekurrieren und - ebenso wie Hamon - einen synekdochischen Volksbegriff vertreten, Macron und Fillon hingegen nicht. ${ }^{24}$ Die Haltung gegenüber dem Volk und diejenige gegenüber den Eliten bedingen sich häufig gegenseitig: Zum einen definiert sich ein synekdochischer Volksbegriff über den Ausschluss mancher Gruppierungen aus dem Volk, was u.a. die Eliten sein können, und zum anderen geht mit der Forderung nach verstärkter politischer Teilhabe des Volkes eine Schwächung der Macht der politischen Eliten einher.

\subsection{Anti-Elitarismus}

Neben dem Rekurs auf das,Volk' ist der Anti-Elitarismus ein konstitutives Element von Populismus (vgl. 3.1). Als Indiz für Anti-Elitarismus kann zunächst die Frequenz von Aussagen, in denen die Eliten einer negativen Wertung unterliegen, fungieren. Tabelle 3 zeigt die Frequenz negativ wertender Prädikationen über die Eliten für die jeweiligen Politiker. Es wird deutlich, dass Mélenchon und Le Pen die Eliten am häufigsten negativ bewerten; Hamon und Fillon tun dies in deutlich geringerem Ausmaß und Macron gar nicht.

\begin{tabular}{ccccc}
\hline Mélenchon & Hamon & Macron & Fillon & Le Pen \\
\hline 11 & 3 & 0 & 2 & 11 \\
\hline
\end{tabular}

Tabelle 3: Frequenz negativ wertender Prädikationen über die Eliten

Aufschluss über den Anti-Elitarismus geben zudem die Ausdrücke, mit denen auf die Eliten referiert wird, da diese Hinweise auf die Konzeptualisierung der Eliten geben und verdeutlichen, wen die jeweiligen Kandidaten als zu den Eliten gehörig befinden. Tabelle

\footnotetext{
${ }^{24}$ Die zentrale Rolle, die das Volk im Diskurs Hamons, Le Pens und Mélenchons einnimmt, korreliert damit, dass dieselben drei Kandidaten unmittelbare Formen politischer Teilhabe propagieren, etwa in Form von Referenden.
} 
4 gibt einen Überblick über einige der von den Kandidaten verwendeten Referenzausdrücke, die im Folgenden erläutert und konkretisiert werden.

\begin{tabular}{lccccc}
\hline & Mélenchon & Hamon & Macron & Fillon & Le Pen \\
\hline élites & 0 & 1 & 0 & 0 & 2 \\
caste & 2 & 0 & 0 & 0 & 0 \\
Eigennamen & 1 & 0 & 0 & 0 & 3 \\
definite Beschreibungen & 8 & 2 & 0 & 2 & 6 \\
\hline
\end{tabular}

Tabelle 4: Referenzausdrücke für das Konzept Eutiten

Mélenchon zeichnet sich durch eine ausgeprägte Eliten-Feindlichkeit aus. Eines seiner Alleinstellungsmerkmale ist, dass er die Eliten als caste bezeichnet (9). Durch diese Bezeichnung wird eine Unüberwindbarkeit gesellschaftlicher Grenzen suggeriert und - durch die negative Wertung, die der Begriff impliziert, - zugleich kritisiert.

(9) Mélenchon: Il est temps d'abolir la monarchie présidentielle et de mettre fin au règne de la CASTE, qui est là, à cheval entre les affaires privées et les affaires de l'État, qui commande à tout, qui le plus souvent est absolument impunie, quoi qu'elle fasse, et qui dirige les affaires générales en fonction de son intérêt.

Teil der Eliten, die Mélenchon ablehnt, sind u.a. die ehemaligen Präsidenten Nicolas Sarkozy und François Hollande, die Mélenchon des Volksverrats bezichtigt:

Mélenchon: Deux présidents français ont trabi la parole du peuple. La première fois ils avaient voté non, il a signé oui, c'est Monsieur Sarkozy. Et le second a dit: „Je vais renégocier les traités budgétaires." Et il n’a rien renégocié du tout.

Hamon vertritt lediglich eine sehr schwache anti-elitäre Haltung. Zwei seiner in Tabelle 3 aufgeführten Aussagen sind lediglich als konstruktive Kritik, nicht aber als Indizien eines Anti-Elitarismus zu werten. In diesen Aussagen bezieht er sich auf die classe politique und die responsables politiques, wobei er sich selbst als Teil dieser politisch Verantwortlichen auffasst und seine Aufgabe darin sieht, zu einer Änderung der aktuellen Situation beizutragen:

(11) Hamon: [...] finalement, on a la classe politique que l'on mérite. Et on aura une démocratie médiocre avec une classe politique médiocre, et il nous revient de prendre nos responsabilités.

In nur einer einzigen Aussage polarisiert Hamon zwischen Volk und Eliten und schwächt zudem die Kritik an den Eliten durch die adverbialen Wendungen un peu und parfois ab:

(12) Pujadas: [...] cette unité nationale, elle est toujours vivace (.) parmi le peuple français.

Hamon: Parmi le peuple. Un peu moins parmi ses élites parfois. 
Fillon trifft zwar zwei negativ wertende Aussagen über Instanzen, die zu den Eliten zählen, jedoch lässt sich ihm keine anti-elitäre Haltung attestieren. Die erste Aussage beinhaltet eine Kritik an der Krankenversicherungspolitik des gouvernement actuel und zeugt damit von einer punktuellen Kritik in Bezug auf ein bestimmtes Thema und nicht von der Konstruktion eines Feindbildes von der Regierung insgesamt. Die zweite Aussage (13) beinhaltet eine Kritik an einer ganzen Generation von Politikern, zu der er sich aber selbst zählt und zu deren Änderung er beitragen möchte. Anlass für seine Kandidatur sei ein Schuldgefühl,

(13) Fillon: [...] le remords d'appartenir à une génération qui n’a pas réussi à faire reculer le chômage et qui a pas réussi à redresser le pays. [...]. J'arrêterai pas ma vie politique sans avoir corrigé ce que je considère à être comme, au fond, des erreurs qui ont été commises par toute une génération de droite comme de gauche.

Le Pen zeichnet sich wie Mélenchon durch einen ausgeprägten Anti-Elitarismus aus. Sie verwendet häufig die Strategie, all ihre politischen Gegner gleichzusetzen. So auch im folgenden Beispiel, in dem sie der, Gesamtheit der Eliten“ vorwirft, dem Volk die Schuld an der hohen Arbeitslosenquote zuzuweisen:

(14) Le Pen: Monsieur Pujadas, je suis encore obligée de contester votre analyse, voilà, eh qui est celle du MEDEF, qui est celle de l'ensemble de nos élites, qui consiste une fois de plus à culpabiliser toujours les Français. [...] Je dis aux Français: arrêtez de vous laissez [culpabiliser, voilà.]

Stattdessen weist Le Pen nicht dem Volk, sondern implizit den Eliten die Schuld zu und konzeptualisiert so die Eliten als Täter, das Volk hingegen als Opfer. So unterstellt sie etwa in (15) den Eliten (ceux qui nous ont gouvernés) ein Verantwortungsbewusstsein ob eines unzureichenden Einsatzes für Sicherheit und gegen Terrorismus und damit auch eine (Mit-)Schuld daran, dass terroristische Attentate stattgefunden haben:

(15) Le Pen: Alors, je comprends que cenx qui nous ont gouvernés ont peut-être un sentiment de responsabilité à l'égard des résultats dans ces domaines de la sécurité et du terrorisme.

Teil der Eliten sind laut Le Pen alle politisch Verantwortlichen in Frankreich (vgl. 14, 15 oder auch l'intégralité de nos dirigeants politiques), aber auch im Ausland. Ihre Feindschaft richtet sich insbesondere gegen Angela Merkel, der sie vorwirft, die Europäische Union im Alleingang zu regieren, und die sie der Demütigung Frankreichs bezichtigt (16). Zudem habe sie Recep Tayyip Erdoğan durch das Flüchtlingsabkommen ermöglicht, Frankreich zu ,erpressen‘:

(16) Le Pen: Non, c'est Madame Merkel qui est allée passer un accord avec Monsieur Erdoğan SANS demander l'avis de personne et encore moins le nôtre. J'ai/ 
j'ai vraiment objectivement beaucoup de mal à supporter la multiplication des bumiliations que subit notre pays euh euh notamment, il faut bien le dire, de la part de Madame Merkel. En faisant cela, Madame Merkel a permis à Monsieur Erdoğan d'avoir euh/ de faire preuve d'un véritable CHANTAGE à notre égard.

Resümierend kann festgehalten werden, dass ein zentrales Merkmal von Populismus darin besteht, die Eliten als Feind zu konzeptualisieren und aus dem Volksbegriff auszugrenzen. Zu den Eliten zählen insbesondere die machthabenden Politiker im In- und Ausland. Charakteristisch für den populistischen Diskurs ist somit die Gegenüberstellung von Volk und Eliten, also deren Konzeptualisierung als Antagonisten. Dass dieser nur einer von vielen Antagonismen ist, die den populistischen Diskurs prägen, macht der folgende Abschnitt deutlich.

\subsection{Antagonismen als Kern populistischer Logik}

Im Folgenden wird gezeigt, dass Antagonismen als Kern populistischer Logik aufgefasst werden können und sich der populistische Diskurs vom politischen Diskurs maßgeblich dadurch unterscheidet, dass erster antagonistisch, letzterer hingegen agonal ist. Zunächst wird diese Abgrenzung theoretisch erörtert und schließlich wird das Korpus auf Antagonismen hin untersucht.

Antagonismus bedeutet ,Gegensatz', wörtlich auch ,Gegnerschaft‘ oder ,Widerstreit‘ (vgl. Duden Online, s.v. Antagonismus; DWDS, s.v. Antagonismus). Dabei steht der Begriff Antagonismus i.d.R. für einen besonders starken, zuweilen unüberbrückbaren Gegensatz; dies indiziert auch das am häufigsten in Verbindung mit Antagonismus auftretende Adjektivattribut unversöhnlich (vgl. DWDS-Wortprofil Antagonismus). Für die Abgrenzung der Begriffe antagonistisch und agonal erweist sich die Etymologie als hilfreich: Beide sind auf gr. agón ả $\gamma \dot{\omega} v$,Kampf(platz), Wettkampf' zurückzuführen (vgl. DWDS, s.v. Antagonismus); sowohl der antagonistische als auch der agonale Diskurs sind also durch Kämpfen ähnelnde, widerstreitende Aushandlungsprozesse gekennzeichnet. Der zentrale Unterschied lässt sich am in antagonistisch enthaltenen Präfix anti-, gegen' festmachen, das darauf hinweist, dass dem Begriff antagonistisch die Bedeutungskomponente des Gegnerischen inhärent ist. Im Gegensatz zum agonalen politischen Diskurs zeichnet sich der antagonistische populistische Diskurs somit dadurch aus, dass Gegenspieler nicht neutral als Akteure eines Aushandlungsprozesses (Agonisten), sondern als Gegner bzw. Feinde (Antagonisten) konzeptualisiert werden.

Die Idee, dass Antagonismen charakteristisch für Populismus sind, wird von zahlreichen Populismusforschern vertreten (vgl. 3). So beginnt etwa Hartlebs (2014, 53) Definition von Populismus mit den Worten „Populismus ist eine antagonistische Erscheinung [...]" und sowohl Hartleb als auch Priester definieren Populismus über dessen AntiWesen: Hartleb $(2014,52)$ bezeichnet Populismus als „eine Art ,Anti-Ismus“" und für Priester (2012,3-4) ist Populismus „kein Substanz, sondern ein Relationsbegriff“, der sich 
„nur in Relation zu einem akuten Gegner bestimmen lässt“. Der antagonistischen Logik liegt ein dichotomisches Prinzip zugrunde. Populisten teilen die Gesellschaft in zwei Lager: Freund vs. Feind oder auch wIR vs. DIE ANDEREN. Im Sinne dieser „Freund-FeindDichotomisierung" (Reisigl 2002, 166) konstruieren Populisten zahlreiche Feindbilder. Der antagonistischen Logik folgend sind die Freunde bzw. Mitglieder der wir-Gruppe die Guten, die Feinde bzw. die anderen hingegen die Bösen. Die Ursache für Probleme suchen Populisten in der Regel beim anderen, der als Sündenbock konzeptualisiert wird oder der Verschwörung bezichtigt wird. Darauf fußen die für den populistischen Diskurs typische Sündenbockrhetorik und die Propagierung von Verschwörungstheorien. Charakteristisch für die antagonistische Logik ist eine manichäische Darstellungsweise, also eine schwarz-weiß-malerische Einteilung der Welt, die mit einer Simplifizierung und Komplexitätsreduktion einhergeht.

Im hier untersuchten Korpus lassen sich folgende Antagonismen konstatieren: Terrorismus/Islamismus vs. westliche Demokratien; Eliten vs. Volk; Journalisten/Medien vs. Politiker; supranationale Organisationen vs. Nationalstaaten; Migranten/Ausländer vs. Inländer; System vs. Alternative. Tabelle 5 gibt einen Überblick über die Antagonisten und die Häufigkeit, mit der die jeweiligen Kandidaten auf die Antagonisten referieren.

\begin{tabular}{lcccccc}
\hline & Mélenchon & Hamon & Macron & Fillon & Le Pen & Gesamt \\
\hline Terrorismus/Islamismus & 3 & 9 & 8 & 27 & 26 & 73 \\
Eliten $^{25}$ & 11 & 1 & 0 & 0 & 11 & 23 \\
Journalisten/Medien & 0 & 0 & 0 & 2 & 8 & 10 \\
Supranationale Organisationen & 2 & 0 & 0 & 0 & 4 & 6 \\
Migranten/Ausländer & 0 & 0 & 0 & 0 & 5 & 5 \\
System & 2 & 0 & 0 & 1 & 0 & 3 \\
andere & 0 & 0 & 3 & 0 & 1 & 4 \\
\hline Gesamt & 18 & 10 & 11 & 30 & 55 & \\
\hline
\end{tabular}

Tabelle 5: Frequenz der Referenz auf Antagonisten

Wie Tabelle 5 zeigt, konstruieren alle Politiker Antagonismen, jedoch in unterschiedlichem Ausmaß. Hervor sticht Le Pen, die nicht nur insgesamt am häufigsten auf Antagonisten referiert, sondern deren Diskurs auch die größte Bandbreite an Feindbildern aufweist. Es folgen, in absteigender Reihenfolge, Fillon, Mélenchon, Macron und Hamon.

\footnotetext{
${ }^{25}$ Hier sind nur diejenigen Referenzen erfasst, in denen die Eliten nicht bloß einer negativen Wertung unterliegen (vgl. Tabelle 3), sondern tatsächlich als Feind konzeptualisiert werden.
} 
Das am häufigsten und ausnahmslos von allen beschworene Feindbild ist der Terrorismus bzw. Islamismus. ${ }^{26}$ Er wird als Antagonist zu Frankreich und anderen westlichen Demokratien sowie im engeren Sinne zu den von ihnen vertretenen Werten konzeptualisiert. In Anbetracht der Tatsache, dass auch die Terroristen sich selbst als Feind konzeptualisieren und dass von ihnen eine reale Gefahr für Frankreich ausgeht, kann die Konzeptualisierung des Terrorismus als Feind nicht als typisch populistisch gewertet werden. Die Benennung des Feindes erfolgt entweder in personifizierter Form mit terroriste(s) oder aber in entpersonifizierter und abstrahierter Form durch die Nennung der für viele Attentate verantwortlichen Organisation (État islamique, Daech) oder des Phänomens als solchem (terrorisme). Fillon und Le Pen - die dieses Feindbild mit Abstand am häufigsten thematisieren - prägen darüber hinaus mit totalitarisme islamiste (17) und fondamentalisme islamiste (18) eigene Begriffe, um die hinter dem Phänomen stehende Ideologie zu bezeichnen. Damit verfolgen sie ein zweifaches Ziel: Zum einen geht damit eine Entpersonifizierung des Feindes einher, durch die der Kampf gegen diesen Feind moralisch leichter fällt, denn die Beseitigung eines Menschen wiegt moralisch schwerer als die einer Ideologie. Zum anderen bewirkt dieses Vorgehen eine Abstrahierung des Feindes, die dazu führt, dass der Feind weniger greifbar und fassbar wird, wodurch er noch bedrohlicher wirkt und die Angst vor ihm geschürt wird.

(17) Fillon: C'est/ c'est ce que moi, j'appelle le totalitarisme islamiste.

(18) Le Pen: Il faut s'attaquer à la racine du mal parce que le terrorisme, c'est l'arme qui est dans la main du fondamentalisme islamiste, qui est une idéologie totalitaire.

Mélenchon unterscheidet sich insofern von den anderen Kandidaten, als dass er als einziger nie die Wörter terrorisme und terroriste(s) verwendet, sondern stattdessen verallgemeinernd von les criminels, les violents und l'ennemi spricht und versucht, die Zuhörer zu beruhigen:

(19) Mélenchon: Pas de panique [...]. [...] notre devoir de citoyen est de ne pas nous abaisser à des polémiques dont rêve l'ennemi, mais au contraire de rester unis.

An zweiter Stelle steht der Antagonismus Eliten vs. Volk (s. dazu ausführlich 5.2). Das Feindbild der Eliten beschwören insbesondere Mélenchon und Le Pen. Mélenchon sticht dadurch hervor, dass er das Volk und die Eliten häufig unmittelbar gegenüberstellt, wodurch der Polarisierung besondere Schärfe verliehen wird (vgl. Belege 1 und 10).

Am dritthäufigsten werden die Medien bzw. Journalisten als Feind konzeptualisiert. Dies ist typisch für viele populistische Bewegungen; man denke nur an das im deutschen

\footnotetext{
${ }^{26}$ Die Wichtigkeit der Thematik ist darauf zurückzuführen, dass Frankreich unter der Präsidentschaft Hollandes mehrfach Ziel schwerer terroristischer Anschläge war, bei denen zahlreiche Menschen ums Leben kamen, weshalb die Franzosen - zum Zeitpunkt der Sendung seit 17 Monaten - unter den Bedingungen des état d'urgence lebten. Das sich während der Sendung ereignende Attentat (vgl. 4) bewirkt nicht nur ein größeres Gewicht, sondern auch eine Emotionalisierung der Thematik in dieser Sendung.
} 
Sprachgebrauch verbreitete politische Schlagwort Lügenpresse, das inzwischen auch Eingang in das Französische gehalten hat. So greift etwa Le Monde explizit den deutschen Ausdruck Lügenpresse auf und glossiert ihn mit „presse mensongère“ (Lemaître 2015). Dieser Kunstgriff erlaubt es den Populisten, die gesamte Berichterstattung pauschal infrage zu stellen und eine eigene Realität zu konstruieren, in der sie selbst über wahr und falsch entscheiden. In diesem Zusammenhang sind auch die Debatte um den Eintritt in ein ,postfaktisches Zeitalter' und die Schlagwörter fake newes und alternative facts zu verorten. ${ }^{27}$ Einen gegen die Medien gerichteten Diskurs führen Fillon und Le Pen. Die Tatsache, dass Fillon die Wahl eines ,medialen Systems` mit der Wahl eines Präsidenten vergleicht (20), zeigt, dass in seiner Vorstellung ein Medium genau wie ein Präsidentschaftskandidat seine eigene Realität, seine eigene Wahrheit konstruiert. Die Aussage, dass nicht die Medien, sondern die Franzosen über den Wahlausgang entscheiden würden, impliziert den Vorwurf an die Medien, diese Entscheidung treffen zu wollen:

(20) Fillon: [L'autorité morale, elle me/] elle/ elle me vient de ma/ de mon passé, elle me vient de 36 ans de vie publique. Euh elle me viendra de/ du CHOIX des Français, voilà. La démocratie, c'est/ c'est pas le choix, comment dirais-je, du système médiatique, c'est le choix des Français. Et c'est ce qui va se passer dimanche, c'est/ dimanche, c'est une autre histoire qui commence.

Le Pens medienfeindliche Haltung kommt darin zum Ausdruck, dass sie die Journalisten falscher Aussagen bezichtigt, was sich in das argumentative Schema der Lügenpresse fügt. Die Journalisten werden hier als Antagonisten zum Sprecher selbst konzeptualisiert, dessen Aussagen die Wahrheit beinhalteten.

(21) Le Pen [zu Salamé]: D’abord, ceci est faux.

(22) Le Pen: Monsieur Pujadas, je suis encore obligée de contester votre analyse, [...]. Tout cela est faux.

An vierter Stellte steht das Feindbild der supranationalen Organisationen. Diese werden als Antagonist zum Nationalstaat, in diesem Fall zu Frankreich, konzeptualisiert. Die Feindschaft gegenüber supranationalen Organisationen steht in engem Zusammenhang mit dem Anti-Elitarismus, da häufig die Eliten die Macht in supranationalen Organisationen innehaben. Zudem werden supranationale Organisationen häufig als Bedrohung der Souveränität und Unabhängigkeit des Nationalstaates wahrgenommen. Darin wurzelt insbesondere Le Pens Feindschaft gegenüber der Europäischen Union, die laut Le

\footnotetext{
${ }^{27}$ Fake newes sind absichtliche Falschmeldungen, wohingegen der Autor alternativer Fakten an das glaubt, was er sagt. Dabei handelt es sich keineswegs um neue Phänomene - als fake newws wurden bereits Ende des 19. Jahrhunderts Falschmeldungen in Zeitungen bezeichnet -, doch ist der Begriff fake news seit dem Wahlkampf Donald Trumps im Herbst 2016 Legion. Den Begriff alternative facts prägte im Januar 2017 dessen Beraterin Kellyanne Conway. Im Französischen findet der Anglizismus fake nerws ebenfalls Verwendung, doch schlägt etwa die Académie française in der Rubrik "Dire, ne pas dire“ vor, anstelle von fake newes die Bezeichnungen bobard, boniments, contre-vérité, mensonge, ragot, tromperie oder trucage zu verwenden (vgl. Académie française 2017). Der englische Terminus alternative facts wurde als faits alternatifs ins Französische entlehnt.
} 
Pen alle nationalen Freiheiten ,verbiete'; so ist das einzige Verb, das Le Pen im Korpus in Verbindung mit der EU verwendet, interdire. Mélenchons Feindschaft richtet sich insbesondere gegen die NATO (OTAN) sowie gegen die staatlichen Zusammenschlüsse G8 und G2O, die er als ,Reichenclubs' diffamiert:

(23) Mélenchon: Je suis donc hostile aux G8, les G20 et tous ces petits clubs de riches qui font la loi dans le monde.

Der Antagonismus Migranten/Ausländer vs. Franzosen wird einzig von Le Pen konstruiert und korreliert mit ihrem auf dem ethnos beruhenden Volkskonzept (vgl. 5.1). Das Feindbild der Migranten bzw. Ausländer ist typisch für rechtspopulistische Bewegungen, wie Decker $\left([2000]^{2} 2004,36\right)$ ausführt und diesbezüglich präzisiert: „Wichtig ist der Zusammenhang von äußerer und innerer Feindlage. Als äußerer und unmittelbar wahrgenommener Feind erscheint in der Regel der Fremde (Nicht-Bürger oder Randständige), als innerer Feind derjenige, der das Eindringen des Fremden betreibt oder geschehen lässt." Der äußere Feind ist bei Le Pen der Ausländer, Immigrant oder Geflüchtete, der in Frankreich bzw. in der EU leben möchte, und der innere Feind sind die Politiker, die dies zulassen, wie etwa Merkel und Hollande (vgl. Beleg 16). Le Pens Ausländerfeindlichkeit kommt in verschiedenen im Wahlkampf vertretenden Forderungen zum Ausdruck, etwa dem Einwanderungsstopp, der sofortigen Ausweisung aller straffällig gewordenen Ausländer (fichiers $S$ ) und der Abschaffung des ius soli (vgl. Beleg 7). Die hohe Zahl der in Europa eintreffenden Migranten bezeichnet sie metaphorisch als Überflutung Europas (24) und konzeptualisiert jene damit als lebensbedrohliche Gefahr, die den Untergang Europas zur Folge habe. Diese Metapher fortspinnend, konzeptualisiert sie die Migranten als Sündenbock, indem sie einen direkten kausalen Zusammenhang zwischen der hohen Zahl einreisender Migranten einerseits und Problemen in Sachen Wirtschaft, Sozialversicherung, innerer Sicherheit und nationaler Einheit andererseits herstellt (25).

(24) Le Pen: Et aujourd'hui, Monsieur Erdoğan menace toutes les semaines de rompre cet accord et de permettre une véritable SUBmersion migratoire de l'Europe $[\ldots]$.

(25) Le Pen: [...] Parce que si non, encore une fois, nous ne POURRons pas arrêter cette vague migratoire qui a des conséquences très lourdes sur notre économie, sur notre système de protection sociale, mais aussi évidemment sur notre sécurité et sur notre unité.

Ein weiteres Feindbild, dessen sich Populisten häufig bedienen, ist das System an sich. Eine solche systemfeindliche Haltung lässt sich im Korpus bei Mélenchon und Fillon konstatieren. ${ }^{28}$ Die zentrale Forderung Mélenchons im Präsidentschaftswahlkampf 2017 ist

\footnotetext{
${ }^{28}$ Im Laufe des Wahlkampfs hat sich auch Macron mehrfach als ,Anti-System-Kandidat' präsentiert, der nicht aus dem System hervorgegangen sei und dieses grundlegend verändern wolle: „La plupart de gens qui sont
} 
die Gründung der Sechsten Republik und die damit verbundene Abschaffung der Fünften Republik, die er als monarchie présidentielle (vgl. Beleg 9) bezeichnet. Damit stellt Mélenchon das aktuelle politische System in seinen Grundsätzen in Frage. Darüber hinaus impliziert dieser Ausdruck eine Kritik am Status des französischen Präsidenten, der mit einem Monarchen verglichen wird, worin eine anti-elitäre Haltung zum Ausdruck kommt. Fillons Systemfeindlichkeit äußert sich z.B. darin, dass er dem System die Schuld an einem Übermaß an Bürokratie gibt:

(26) Fillon: [...] il y a [...] un excès de bureaucratie. Et c'est pas les fonctionnaires qui en sont responsables [...]. C’est le système qui a été mis en place.

Unter der Kategorie „andere“ in Tabelle 5 sind der Rekurs Le Pens auf die Arbeitgebervereinigung MEDEF (Mouvement des Entreprises de France, vgl. Beleg 14) sowie vier Aussagen Macrons resümiert. In diesen vier Aussagen konzeptualisiert Macron Antagonisten, ohne sie jedoch beim Namen zu nennen. In der Regel handelt es sich dabei um Personen - vermutlich Politiker und/oder Journalisten -, deren Haltung in Bezug auf eine bestimmte Thematik der seinen entgegengesetzt ist und die er der Falschaussage oder Verleumdung bezichtigt. So wirft er ihnen etwa in (27) vor, seine Reform des ISF (impôt sur la fortune) wissentlich falsch darzustellen, und in (28), seine Aussage über die französische Kultur ${ }^{29}$ zu deformieren. Durch die Strategie, die Gegner nicht beim Namen zu nennen, bietet er diesen einerseits weniger Angriffsfläche, hebt aber andererseits auch den Kampf von einer persönlichen auf eine sachliche Ebene.

Macron: Donc, toutes les fausses rumeurs là-dessus, c'est de la calomnie.

(28) Macron: Et donc, j'ai dit/ ce que j'ai dit dans cette discussion, qui a été citée abondamment, déformée et calomniée, c'est que [...].

Abschließend sei neben dem Terrorismus (s. supra) ein weiterer Aspekt genannt, anhand dessen sich der Unterschied zwischen dem populistischen und dem politischen Diskurs verdeutlichen lässt. Selbstverständlich manifestiert sich im Korpus auch ein Kampf zwischen den Präsidentschaftskandidaten, die im Wahlkampf gegeneinander antreten und somit direkte Konkurrenten sind. Die einzelnen Kandidaten konzeptualisieren ihre jeweiligen Gegenkandidaten zumeist als Mitbewerber oder Konkurrenten, jedoch in der Regel nicht als Feinde. Dies ist als Manifestation der Agonalität des politischen Diskurses zu werten, die im Wahlkampf besondere Schärfe gewinnen kann, jedoch nur in Aus-

\footnotetext{
autour de moi sont dans la vie politique depuis des années, des décennies. [...] Ils ont jamais renouvelé la vie politique et ils préfèrent que ça n'arrive pas. Moi, j’ai lancé un mouvement politique qui la renouvelle vraiment.“ (Macron in Le Grand Débat I); „Je crois pour ma part que notre pays aujourd'hui, il est bloqué par un système politique qui ne veut plus des vraies réformes. [...] Eh bien, ce que je vous propose, c'est l'alternance véritable, profonde, le renouvellement de notre vie politique (.) complet [...]." (Macron in Le Grand Débat II).

${ }^{29}$ Anlässlich eines Wahlkampfauftritts hatte Macron den Satz „Il n’y a pas une culture française“ geäußert, der eine große Polemik auslöste (Macron 2017, vgl. Anm. 30 für seine nachträglich geäußerte Erläuterung des Satzes).
} 
nahmefällen Dimensionen der antagonistischen Logik des populistischen Diskurses annimmt.

Resümierend lässt sich festhalten, dass sich die zentrale Bedeutung der antagonistischen Logik für den populistischen Diskurs in einer manichäischen Freund-Feind-Dichotomisierung manifestiert und mit der Konstruktion zahlreicher Feindbilder und Mechanismen der Aus- und Abgrenzung einhergeht. Als antagonistische Erscheinung geht der Populismus damit deutlich über die Agonalität des politischen Diskurses hinaus. Der Übergang zwischen beiden ist freilich fließend, weshalb im Folgenden die Analyseergebnisse in einer Synthese, die es vermag, den Facettenreichtum des Phänomens des Populismus zu erfassen, zusammengeführt werden sollen.

\subsection{Spielarten des Populismus}

Die Analyse hat gezeigt, dass der Sprachgebrauch aller untersuchten Akteure populistische Merkmale aufweist, da jeder Akteur zumindest manche der untersuchten Kriterien erfüllt. Jedoch muss unterschieden werden, welche Kriterien in welcher Kombination und in welcher Intensität auftreten. Notwendige Kriterien für das Vorhandensein eines genuinen Populismus sind das synekdochisch konzeptualisierte Volk, der AntiElitarismus und die antagonistische Logik. Die Kriterien 1.1 und 2.1 - häufiger Rekurs auf das Volk und negativ wertende Aussagen über die Eliten - sind alleine nicht hinreichend, um von Populismus sprechen zu können. Die antagonistische Logik kommt nicht nur in der Gegenüberstellung von Volk und Eliten, sondern auch in weiteren Antagonismen zum Ausdruck. Zentral ist, dass die Antagonisten in einer manichäischen, dichotomischen Darstellungsweise als Feinde gegenübergestellt werden; darin unterscheidet sich der antagonistische populistische Diskurs von dem agonalen politischen Diskurs. Zeugt der Diskurs eines Politikers von einer antagonistischen Logik, aber weder von einem synekdochischen Volksbegriff noch von Anti-Elitarismus, so kann nicht von einem genuinen Populismus, sondern lediglich vom Vorhandensein einer populistischen Logik die Rede sein.

In Abhängigkeit davon, welche Kriterien in welcher Kombination und Intensität auftreten, lassen sich verschiedene Spielarten von Populismus unterscheiden. Zunächst ist grundlegend an der traditionellen Unterscheidung von Links- und Rechtspopulismus festzuhalten: Linkspopulismus liegt ein inklusives, auf einer bestimmten sozialen ,Schicht beruhendes Volkskonzept zugrunde, Rechtspopulismus hingegen in exklusives, auf dem ethnos beruhenden Volkskonzept. Le Pen kann als Vertreterin des Rechtpopulismus par excellence gelten, nicht zuletzt aufgrund ihres über das ethnos definierten Volksbegriffs. Als Linkspopulisten können Mélenchon und Hamon gelten. Allerdings erweist sich Mélenchon als deutlich populistischer als Hamon, da er häufiger auf das Volk rekurriert, häufiger gegen Eliten polarisiert und auch mehr Feindbilder konstruiert. Fillon ließe sich nicht als Vertreter eines genuinen Populismus, sondern vielmehr als Vertreter eines ,Wahlkampfpopulismus‘ oder, mit Decker und Lewandowsky (2011, 346-348), eines „Mainstream-Populismus“ klassifizieren. Dieser ist dadurch charakterisiert, dass 
kein synekdochischer Volksbegriff und kein Anti-Elitarismus, wohl aber eine antagonistische Logik in Bezug auf andere Gegensatzpaare auftritt. Im Fall Macrons ließe sich diskutieren, ob er nicht sogar als Antipopulist bezeichnet werden könnte. Dafür spricht, dass er weder einen synekdochischen Volksbegriff noch eine anti-elitäre Haltung vertritt und auch wenig antagonistisch, sondern im Gegenteil häufig gezielt pluralistisch ${ }^{30}$ und anti-polarisierend, konsensorientiert und sachlich agiert. ${ }^{31}$ Allerdings kann gerade dieses anti-polarisierende Verhalten auch als Strategie aufgefasst werden, die dazu dient, pluralistische Meinungsbildung zu verhindern und Kritik gar nicht erst den Raum zu geben zu entstehen. So bestimmt Macron z.T. darüber, welche Medien und Journalisten bei bestimmten Ereignissen anwesend sein dürfen, und die von ihm gegründete Partei LREM übertüncht als Partei der Mitte und durch das Prinzip der double appartenance politische Gegensätze, von denen aber die Demokratie lebt. Auch Macrons Selbstinszenierung als Anti-System-Kandidat und einige sprachliche Fauxpas könnten mit populistischen Strategien in Verbindung gebracht werden. Die Frage, was unter Antipopulismus zu verstehen ist und ob Macron als Antipopulist bezeichnet werden kann, verdient eine ausführlichere Betrachtung in einer weiteren Studie.

Abb. 1 visualisiert das Verhältnis, in dem die untersuchten Kandidaten als Vertreter der verschiedenen Spielarten von Populismus zueinander stehen. Die Einordnung der Akteure basiert auf zwei Kontinua, dem politisch-ideologischen Spektrum von links nach rechts auf der $\mathrm{x}$-Achse und der Intensität der Ausprägung des Populismus auf der y-Achse.

\footnotetext{
${ }^{30}$ Dies zeigt etwa das von ihm vertretene Konzept einer hybriden Identität, das für Offenheit, Vielfalt und Veränderbarkeit steht und grundlegend für seine Haltung gegenüber Globalisierung und Integration ist: „Donc, la culture française, elle existe, mais dans cette diversité, cette richesse et ce caractère INDOMPTABLE que je défendrai toujours. Et donc, il n'y a pas UNE culture française, comme certains voudraient l'enfermer, comme UNE identité française qui serait une identité du même. Il y a une identité ouverte qui est avant tout un projet."

31 Macron zeigt häufig Gemeinsamkeiten zwischen den Programmen verschiedener Kandidaten auf und fordert seine Anhänger zu einem respektvollen Umgang mit den Gegenkandidaten auf, wie sein regelmäßig bei Wahlkampfauftritten geäußertes Ne les sifflez pas! mustergültig illustriert. Auch schürt er keine Ängste, sondern spricht stattdessen von défis und transformations, denen man sich gerne stelle.
} 


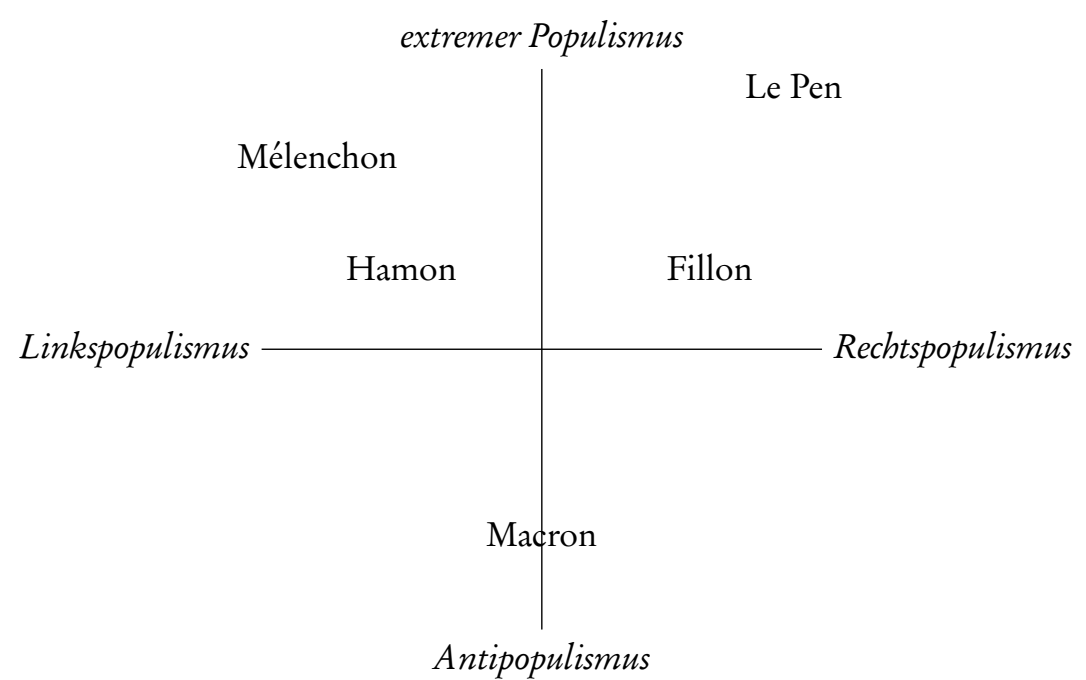

Abbildung 1: Visualisierung verschiedener Spielarten von Populismus

Abb. 1 ermöglicht eine hilfreiche Visualisierung der Ergebnisse, deren Verdienst insbesondere in einer adäquaten Darstellung des Phänomens als Kontinuum besteht. Dies betrifft sowohl die Einordnung im ideologischen Spektrum von links nach rechts als auch die Intensität der Ausprägung von Populismus, die auf der Frequenz der untersuchten Kriterien beruht. Allerdings geht die Visualisierung auch mit einer Generalisierung und einem Informationsverlust einher. So geht z.B. aus der Graphik nicht hervor, dass Hamon einen synekdochischen Volksbegriff und eine anti-elitäre Haltung vertritt, Fillon hingegen nicht, letzterer dafür aber mehr Antagonismen konstruiert als ersterer. Auch der Unterschied zwischen Le Pen, die sich als Sprachrohr des Volkes inszeniert, und Mélenchon, der sich vielmehr als Teil des Volkes versteht, verschwindet hinter der Information, dass für beide das Volk von äußerster Wichtigkeit ist. Die Graphik ist darüber hinaus recht schematisierend; eine genaue Einordnung, die eine Skalierung der Achsen erfordern würde, wurde nicht vorgenommen. Dafür wäre eine Quantifizierung der Ergebnisse nötig, wie sie etwa Jagers und Walgrave (2007) und Scharloth (2017) unternommen haben (vgl. 3.2). Eine solche Quantifizierung der Ergebnisse zur Errechnung eines Populismus-Index hat sicherlich Vorteile, ist jedoch auch mit Schwierigkeiten verbunden. Zum einen müssen zunächst haltbare Kriterien geschaffen werden, deren Auswahl und Gewichtung zumeist diskutabel ist, und zum anderen kann ein rein quantitativer Populismus-Index nur beschränkt qualitative Informationen transportieren, etwa darüber, welche Kriterien in welcher Kombination erfüllt werden. In der vorliegenden Arbeit wird daher einem zu errechnenden Index eine schematisierende Visualisierung vorgezogen. 


\section{Fazit}

Im Zuge des in den letzten Jahren zu konstatierenden inflationären Gebrauchs des Begriffs Populismus - sowohl auf analytischer, metapolitischer bzw. linguistischer Ebene als auch in der politischen Auseinandersetzung selbst - werden die verschiedensten Politiker und Parteien in zahlreichen Ländern als Populisten bzw. populistisch klassifiziert. Setzt man sich mit dem Populismus als scheinbarem Erfolgsmodell der Politik unserer Zeit auseinander, so gilt es zunächst zu klären, was unter Populismus zu verstehen ist. In der vorliegenden Untersuchung wurde der Versuch einer Minimaldefinition unternommen, der zufolge sich Populismus zugleich über eine inhaltliche und eine formale Dimension bestimmen lässt: Populismus ist eine auf einer antagonistischen Logik beruhende Erscheinung, in deren Zentrum der Rekurs auf das synekdochisch konzeptualisierte Volk steht, dem die als feindlich begriffenen Eliten gegenüberstellt werden. Diese konstitutiven Merkmale können je nach Kontext in Kombination mit weiteren ergänzenden Charakteristika auftreten. Der populistische Diskurs unterscheidet sich insofern vom politischen Diskurs, als dass letzterer agonal, ersterer hingegen antagonistisch ist, d.h. Gegenspieler als Feinde konzeptualisiert.

Die Anwendung dieser Kriterien auf die Spitzenkandidaten des französischen Präsidentschaftswahlkampfs 2017 hat gezeigt, dass alle untersuchten Politiker populistische Kommunikationsstrategien und entsprechende Versprachlichungsformen (Referenzausdrücke, implizite und explizite Prädikationen, Argumentationsmuster) verwenden. Allerdings erfüllen die jeweiligen Politiker verschiedene Merkmale populistischen Sprachgebrauchs, die dadurch auch in unterschiedlicher Kombination und zudem in unterschiedlicher Intensität auftreten. Auf dieser Grundlage können verschiedene Spielarten von Populismus unterschieden werden, wobei die herausgearbeiteten Typen Rechtspopulismus, Linkspopulismus, Mainstreampopulismus und Antipopulismus jeweils noch einmal binnendifferenziert und zudem durch weitere Spielarten des Populismus ergänzt werden müssen. Grundlegend ist an der von Puhle bereits 1986 vorgeschlagenen Unterscheidung zwischen genuin populistischen Parteien und Bewegungen und gelegentlich auftretenden „populistische[n]Elemente[n]“, die insbesondere zu Zeiten des Wahlkampfs manifest werden können, festzuhalten (Puhle 1986, 14, 29, 32, vgl. auch Januschek und Reisigl 2014). Somit sind politischer und populistischer Sprachgebrauch nicht binär, sondern als Kontinuum zu konzeptualisieren, in dem sich einzelne Politiker und Parteien je nach Kontext immer wieder neu verorten lassen. Die Tatsache, dass gerade im Wahlkampf häufig zu populistischen Mitteln gegriffen wird, lässt vermuten, dass Populismus als förderlich für den Wahlsieg betrachtet wird. Falls dem so sein sollte - obwohl der Populismus in Frankreich im Jahr 2017 doch nicht den Sieg davongetragen hat -, ist er, wenn überhaupt, nur einer von vielen in einem komplexen Verhältnis zueinander stehenden Faktoren. 


\section{Literatur}

Aalberg, Toril, Frank Esser, Carsten Reinemann, Jesper Stromback und Claes De Vreese, Hrsg. 2017. Populist Political Communication in Europe. New York/London: Routledge.

Académie française. 2017. „Fake news.” 04.05.2017. http://www.academie-francaise.fr/fake-news. Alduy, Cécile. 2017. Ce qu'ils disent vraiment. Les politiques pris aux mots. Paris: Seuil.

Angermuller, Johannes, Martin Nonhoff, Eva Herschinger, Felicitas Macgilchrist, Martin Reisigl, Juliette Wedl, Daniel Wrana und Alexander Ziem, Hrsg. 2014. Diskursforschung. Ein interdisziplinäres Handbuch. Bielefeld: transcript.

Anthony, Laurence. 2016. „AntConc.“ Version 3.4.4, Computer Software. Tokyo, Japan: Waseda University. http://www.laurenceanthony.net/software.

Big Browser. 2017. „L'assassinat politique‘, la métaphore de trop de François Fillon.“ Le Monde, 02.03.2017. http://www.lemonde.fr/big-browser/article/2017/03/02/1-assassinat-politiquela- metaphore-de-trop-de-francois-fillon ${ }_{5} 088435_{4} 832693$.html.

Brugman, Hennie und Albert Russel. 2004. „Annotating Multi-media/Multi-modal Resources with ELAN." In Proceedings of the Fourth International Conference on Language Resources and Language Evaluation (LREC 2004), hrsg. von Maria Teresa Lino, Maria Francisca Xavier, Fátima Ferreira, Rute Costa und Raquel Silva, 2065-2068. Paris: European Language Research Association.

Bühler, Karl. 1934. Sprachtheorie. Die Darstellungsfunktion der Sprache. Jena: Fischer.

Canovan, Margaret. 1981. Populism. London: Junction Books.

Constitution = Assemblée nationale. 1958. Constitution de la République française. Constitution du 4 octobre 1958. Version mise à jour en janvier 2015. http://www.assemblee-nationale.fr/ connaissance/constitution.asp.

Coseriu, Eugenio. 1955-56. „Determinación y entorno. Dos problemas de una lingüística del hablar.“ Romanistisches Jabrbuch 7 (1): 29-54.

Coseriu, Eugenio. (1980) ${ }^{3}$ 1994. Textlinguistik. Eine Einführung, hrsg. von Jörn Albrecht. Tübingen/Basel: Francke.

Coseriu, Eugenio. (1988) 2007. Sprachkompetenz. Grundzüge der Theorie des Sprechens. Tübingen: Narr.

CPC = République française. 2018. „Code de procédure civile.“ Version consolidée au 1 janvier 2018. http://www.legifrance.gouv.fr/affichCode.do?cidTexte=LEGITEXT000006070716\&dat eTexte $=20180611$.

Decker, Frank. $(2000)^{2}$ 2004. Der neue Rechtspopulismus. Opladen: Leske + Budrich.

Decker, Frank. 2006a. „Die populistische Herausforderung. Theoretische und ländervergleichende Perspektiven. “ In Populismus. Gefahr für die Demokratie oder nützliches Korrektiv?, hrsg. von Frank Decker, 9-32. Wiesbaden: Springer VS Verlag für Sozialwissenschaften.

Decker, Frank, Hrsg. 2006b. Populismus. Gefahr für die Demokratie oder nützliches Korrektiv? Wiesbaden: Springer VS Verlag für Sozialwissenschaften.

Decker, Frank und Marcel Lewandowsky. 2011. „Populismus. Erscheinungsformen, Entstehungshintergründe und Folgen eines politischen Phänomens.“ In Das Populäre. Untersuchungen zu Interaktionen und Differenzierungsstrategien in Literatur, Kultur und Sprache, hrsg. von Olivier Agard, 331-351. Göttingen: V\&R/Bonn University Press.

Diehl, Paula. 2012. „Populismus und Massenmedien.“ Aus Politik und Zeitgeschichte 5-6: 16-22. http://www.bpb.de/apuz/75854/populismus-und-massenmedien. 
Duden Online = Dudenredaktion. 2018. Duden Online. Berlin: Bibliographisches Institut GmbH. http://www.duden.de.

DWDS = Berlin-Brandenburgische Akademie der Wissenschaften, Hrsg. o.J. Digitales Wörterbuch der deutschen Sprache. http://www.dwds.de/wb/.

ELAN = The Language Archive. 2017. „ELAN.“ Version 5.0, Computer Software. Nijmegen: Max Planck Institute for Psycholinguistics. http://tla.mpi.nl/tools/tla-tools/elan/.

Esser, Frank, Agnieszka Stępińska und David Nicolas Hopmann. 2017. „Populism and the Media: Cross-National Findings and Perspectives." In Populist Political Communication in Europe, hrsg. von Toril Aalberg, Frank Esser, Carsten Reinemann, Jesper Stromback und Claes De Vreese, 365-380. New York/London: Routledge.

France 2. 2017. „15 minutes pour convaincre.“ 20.04.2017, Video, 03:56:14. http://www.youtube. com/watch?v $=1 R 5 \mathrm{NR} 2 \mathrm{YqV}_{\mathrm{V}} 4$.

Freeden, Michael. 1996. Ideologies and Political Theory. A Conceptual Approach. Oxford: Oxford University Press.

Girnth, Heiko. (2002) ${ }^{2}$ 2015. Sprache und Sprachverwendung in der Politik. Berlin/Boston: De Gruyter.

Grand Robert = Dictionnaires Le Robert. 2017. Le Grand Robert de la langue française. Version 4.1. Paris: Le Robert. http://gr.bvdep.com/robert.asp.

Hartleb, Florian. 2014. Internationaler Populismus als Konzept. Zwischen Kommunikationsstil und fester Ideologie. Baden-Baden: Nomos.

Ionescu, Ghița und Ernest Gellner, Hrsg. 1969. Populism. Its Meaning and National Characteristics. London: Macmillan.

Jagers, Jan und Stefaan Walgrave. 2007. „Populism as Political Communication Style: An Empirical Study of Political Parties' Discourse in Belgium. “ European Journal of Political Research 46 (3): 319-345.

Januschek, Franz und Martin Reisigl. 2014. „Populismus in der digitalen Mediendemokratie - Editorial." Osnabrücker Beiträge zur Sprachtheorie 86 (1): 7-17.

Koch, Peter. 1997. „Diskurstraditionen. Zu ihrem sprachtheoretischen Status und ihrer Dynamik.“ In Gattungen mittelalterlicher Schriftlichkeit, hrsg. von Barbara Frank, Thomas Haye und Doris Tophinke, 43-79. Tübingen: Narr.

Laclau, Ernesto. (2005) 2007. On Populist Reason. London: Verso.

La République En Marche! o.J. „La République En Marche! Le mouvement fondé par Emmanuel Macron." http://www.en-marche.fr.

Lebsanft, Franz. 2015. „Zur Linguistik des populistischen Diskurses. Analyse eines Interviews mit Marine Le Pen (2014).“ In Romanica et Balcanica. Wolfgang Dabmen zum 65. Geburtstag, hrsg. von Tede Kahl, Johannes Kramer und Elton Prifti, 289-301. München: Akademische Verlagsgesellschaft.

Lebsanft, Franz. 2018. „Sprache und Politik. Zu Theorie und Methode der linguistischen Analyse politischer Diskurse." In Wablkampf ist Wortkampf. Präsidentschaftswablkampagnen aus sprachwissenschaftlicher Sicht, hrsg. von Sandra Issel-Dombert und Aline Wieders-Lohéac, 1745. Frankfurt am Main et al.: Peter Lang.

Lebsanft, Franz und Angela Schrott. 2015a. „Diskurse, Texte, Traditionen.“ In Diskurse, Texte, Traditionen. Modelle und Fachkulturen in der Diskussion, hrsg. von Franz Lebsanft und Angela Schrott, 11-46. Göttingen: V\&R/Bonn University Press. 
Lebsanft, Franz und Angela Schrott, Hrsg. 2015b. Diskurse, Texte, Traditionen. Modelle und Fachkulturen in der Diskussion. Göttingen: V\&R/Bonn University Press.

Le Grand Débat I = TF1/LCI/France 24. 2017. „Le Grand Débat.“ 20.03.2017, Video, 03:12:54. https://www.youtube.com/watch?v= VYXhy7Om0gs.

Le Grand Débat II = BFM TV/CNews/RMC/Dailymotion. 2017. „Le Grand Débat.“ 04.04.2017, Video, 03:50:48. https://www.youtube.com/watch?v=OhWRT3PhMJs.

Lemaitre, Frédéric. 2015. „Allemagne: le leader du mouvement anti-islam Pegida se met en retrait." Le Monde, 21.01.2015. http://www.lemonde.fr/europe/article/2015/01/21/allemagne-lelead er-du-mouvement-anti-islam-pegida-se-met-en-retrait ${ }_{4} 560813_{3} 214 . h t m l$.

Macron, Emmanuel. 2017. „Rassemblement à Lyon.“ 04.02.2017, Video, 01:43:26. https://www.you tube.com/watch?v=jZ-wPEOomM.

Mélenchon, Jean-Luc. 2010. „Mélenchon: ,Populiste, moi? J’assume!““ L’Express, 16.09.2010. http: //www.lexpress.fr/actualite/politique/melenchon-populiste-moi-j-assume_919603.html.

Mény, Yves und Yves Surel. 2002. „The Constitutive Ambiguity of Populism.“ In Democracies and the Populist Challenge, hrsg. von Yves Mény und Yves Surel, 1-21. Basingstoke: Palgrave.

Meyer, Thomas. 2006. „Populismus und Medien.“ In Populismus. Gefahr für die Demokratie oder nützliches Korrektiv?, hrsg. von Frank Decker, 81-96. Wiesbaden: VS Verlag für Sozialwissenschaften.

Ministère de l'Intérieur. 2017. „Résultats de l'élection présidentielle 2017.“ http://www.interieur. gouv.fr/Elections/Les-resultats/Presidentielles/elecresult_presidentielle-2017/(path)/presiden tielle-2017/FE.html.

Montvalon, Jean-Baptiste de. 2017. „La ,rencontre d'un homme et d'un peuple‘. Histoire d'un mythe. "Le Monde, 07.03.2017. http://www.lemonde.fr/election-presidentielle-2017/article/2017/ 03/07/la-rencontre-d-un-homme-et-d-un-peuple-histoire-d-un-mythe_5090440_4854003.html.

Mudde, Cas. 2004. „The Populist Zeitgeist.“ Government and Opposition 39 (4): 541-563.

Mudde, Cas und Cristóbal Rovira Kaltwasser. 2012. „Populism and (Liberal) Democracy. A Framework for Analysis." In Populism in Europe and the Americas. Threat or Corrective for Democracy?, hrsg. von Cas Mudde und Cristóbal Rovira Kaltwasser, 1-26. Cambridge: Cambridge University Press.

Priester, Karin. 2007. Populismus. Historische und aktuelle Erscheinungsformen. Frankfurt am Main: Campus.

Priester, Karin. 2012. „Wesensmerkmale des Populismus.“ Aus Politik und Zeitgeschichte 5-6: 3-9. http://www.bpb.de/apuz/75848/wesensmerkmale-des-populismus.

Priester, Karin. 2017. „Das Syndrom des Populismus.“ 16.01.2017. http://www.bpb.de/politik/ extremismus/rechtspopulismus/240833/das-syndrom-des-populismus.

Puhle, Hans-Jürgen. 1986. „Was ist Populismus?“ In Populismus und Aufklärung, hrsg. von Helmut Dubiel, 12-32. Frankfurt am Main: Suhrkamp.

Reisigl, Martin. 2002. „,Dem Volk aufs Maul schauen, nach dem Mund reden und Angst und bange machen'. Von populistischen Anrufungen, Anbiederungen und Agitationsweisen in der Sprache österreichischer PolitikerInnen. “In Rechtspopulismus. Österreichische Krankheit oder europäische Normalität?, hrsg. von Wolfgang Eismann, 149-198. Wien: Czernin.

Reisigl, Martin. 2012. „Zur kommunikativen Dimension des Rechtspopulismus.“ In Populismus. Herausforderung oder Gefahr für die Demokratie?, hrsg. von Sir Peter Ustinov Institut, 141-162. Wien: New Academic Press. 
Rigat, Françoise. 2010. „Mes chers compatriotes': stratégies discursives de l'interpellation des électeurs dans les professions de foi." CORELA - Numéros thématiques. L'interpellation. http: //09.edel.univ-poitiers.fr/corela/index.php?id=783.

Saussure, Ferdinand de. (1916) 2013. Cours de linguistique générale. Zweisprachige Ausgabe frz.-dt. mit Einleitung, Anmerkungen \& Kommentar von Peter Wunderli. Tübingen: Narr.

Scharloth, Joachim. 2017. „Ist die AfD eine populistische Partei? Eine Analyse am Beispiel des Landesverbandes Rheinland-Pfalz.“ Aptum 13 (1): 1-15.

Schlieben-Lange, Brigitte. 1983. Traditionen des Sprechens. Elemente einer pragmatischen Sprachgeschichtsschreibung. Stuttgart: Kohlhammer.

Schrott, Angela. 2015. „Kategorien diskurstraditionellen Wissens als Grundlage einer kulturbezogenen Sprachwissenschaft.“ In Diskurse, Texte, Traditionen. Modelle und Fachkulturen in der Diskussion, hrsg. von Franz Lebsanft und Angela Schrott, 115-146. Göttingen: V\&R/Bonn University Press.

Taggart, Paul A. 2000. Populism. Buckingham/Philadelphia: Open University Press.

Taguieff, Pierre-André. 1997. „Le populisme et la science politique. Du mirage conceptuel aux vrais problèmes." Vingtième Siècle. Revue d'Histoire 56 (4): 4-33.

Taguieff, Pierre-André. 2002. L'illusion populiste. De l'archaïque au médiatique. Paris: Berg.

TLFi $=$ ATILF/CNRS und Université de Lorraine. o.J. „Trésor de la langue française informatisé.“ http://atilf.fr/tlfi.

Wiles, Peter. 1969. „A Syndrome, Not a Doctrine.“ In Populism. Its Meaning and National Characteristics, hrsg. von Ghița Ionescu und Ernest Gellner, 166-179. London: Macmillan.

Wilhelm, Raymund. 2001. „Diskurstraditionen.“ In Sprachtypologie und sprachliche Universalien. Ein internationales Handbuch, hrsg. von Martin Haspelmath, Ekkehard König, Wulf Oesterreicher und Wolfgang Raible, Bd. 1, 467-477. Berlin/New York: De Gruyter.

Worsley, Peter. 1969. „The Concept of Populism.“ In Populism. Its Meaning and National Characteristics, hrsg. von Ghiţa Ionescu und Ernest Gellner, 212-250. London: Macmillan.

Wrana, Daniel, Hrsg. 2014. DiskursNetz. Wörterbuch der interdisziplinären Diskursforschung. Berlin: Suhrkamp. 